\title{
Rotational dynamics of charged colloidal spheres: Role of particle interactions
}

\author{
Gijsberta H. Koenderink ${ }^{\text {a) }}$ \\ Van't Hoff Laboratory for Physical and Colloid Chemistry, Debye Institute, Utrecht University, Padualaan \\ 8, 3584 CH Utrecht, The Netherlands \\ M. Pavlik Lettinga \\ Van't Hoff Laboratory for Physical and Colloid Chemistry, Debye Institute, Utrecht University, Padualaan \\ 8, 3584 CH Utrecht, The Netherlands and Institut Weiche Materie, Institut für Festkörperforschung, \\ Forschungszentrum Jülich, D-52425 Jülich, Germany
}

Albert P. Philipse

Van't Hoff Laboratory for Physical and Colloid Chemistry, Debye Institute, Utrecht University, Padualaan 8, 3584 CH Utrecht, The Netherlands

(Received 10 June 2002; accepted 31 July 2002)

\begin{abstract}
Time-resolved phosphorescence anisotropy (TPA) is used to measure the short-time rotational diffusion coefficient $D_{s}^{r}(\phi)$ of charged tracer spheres as a function of the volume fraction $\phi$ of like-charged colloidal host spheres in nonaqueous solvents. Sphere interactions are varied from long-range repulsive to short-range attractive by changing the ionic strength and the solvent composition. It is shown that $D_{s}^{r}(\phi)$ is very sensitive to details of the interaction near contact, in agreement with theory. In contrast, the low-shear viscosity $\eta_{L}(\phi)$ of the host dispersions is mostly controlled by the tail of the interaction potential. We discuss the applicability of Stokes-EinsteinDebye scaling $D_{s}^{r}(\phi) \propto 1 / \eta_{L}(\phi)$, and $D_{s}^{r}(\phi) \propto 1 / \eta_{\infty}(\phi)$, where $\eta_{\infty}$ is the high-frequency-limiting viscosity. Scaling with $\eta_{L}(\phi)$ fails at high particle and low salt concentrations, while scaling with $\eta_{\infty}$ is fairly good, in particular when an apparent nonstick boundary condition is imposed on the friction factor. We conclude that TPA is well suited for use as a microrheological technique.
\end{abstract}

(C) 2002 American Institute of Physics. [DOI: 10.1063/1.1509054]

\section{INTRODUCTION}

Translational self-diffusion in concentrated colloidal sphere suspensions has been investigated extensively by simulations, experiment, and theory. ${ }^{1}$ In contrast, studies on rotational diffusion in dense sphere suspensions are mostly limited to short-time rotational diffusion in monodisperse hard-sphere suspensions. Well-known techniques to measure rotational diffusion are depolarized dynamic light scattering $(\mathrm{DDLS})^{2,3}$ and nuclear magnetic resonance. ${ }^{4-7}$ Unfortunately, both techniques require special tracer colloids that are difficult to synthesize. A few experiments have been done with forced Rayleigh scattering, ${ }^{8}$ fluorescence photobleaching recovery, ${ }^{9}$ and time resolved phosphorescence anisotropy (TPA). ${ }^{10-12}$ These luminescence-based methods rely on dyelabeled silica or latex tracer particles, which are relatively easy to synthesize.

In this work we study experimentally the dependence of short-time rotational sphere diffusion on the interactions between the rotating tracer and the surrounding host colloids, using TPA (cf. Sec. II B for an explanation of the technique). This extends our previous combined experimental and theoretical study of rotational diffusion in binary sphere mixtures. ${ }^{12}$ The colloidal model system used consists of eosin-labeled silica tracer spheres dispersed in nonaqueous

\footnotetext{
a) Author to whom correspondence should be addressed. Electronic mail: g.h.koenderink@chem.uu.nl
}

suspensions of like-charged silica host spheres. The range of the electrostatic double-layer repulsions is varied by changing the ionic strength. Attractions are induced by adding salt or changing the solvent composition. The effects of particle interactions on rotational diffusion are interpreted using theory for hard and charged spheres (cf. Sec. II A).

We compare our rotational diffusion data with the lowshear viscosity $\eta_{L}$ of the host dispersions. It will be shown that rotational diffusion is more sensitive to details of the interactions near contact than the viscosity, which is commonly used for characterization of particle interactions. ${ }^{13}$ Further, we test whether the tracer rotational diffusion coefficient $D_{s}^{r}$ scales with $1 / \eta_{L}$. For a single free tracer sphere with radius $a_{T}$ in a solvent with shear viscosity $\eta_{0}$ there is an exact relation between rotational diffusion and viscosity, namely the Stokes-Einstein-Debye (SED) relation ${ }^{14-16}$

$$
D_{0}^{r}=\frac{k_{B} T}{8 \pi \eta_{0} a_{T}^{3}},
$$

with $k_{B} T$ the thermal energy. A similar, so-called StokesEinstein (SE) relation holds for the translational diffusion coefficient of a tracer particle in solvent ${ }^{14,15}$

$$
D_{0}^{t}=\frac{k_{B} T}{6 \pi \eta_{0} a_{T}} .
$$


Whether these relations can be generalized to tracer diffusion in dense colloidal host fluids is a much debated issue. If such an extension is possible, tracer dynamics can be used to do microrheology.$^{17}$ Compared to conventional rheology, tracer diffusion measurements, such as TPA, employ relatively small sample volumes, are noninvasive, and can measure local viscosities in inhomogeneous samples. Translational diffusion of hard spheres indeed has a similar (though not identical) concentration dependence as the inverse viscosity ${ }^{18-22}$ but for charged spheres deviations from SE scaling were found. ${ }^{22,24}$ Recently, we showed experimentally and theoretically that rotational diffusion of hard spheres also has a similar, but again not identical, concentration dependence as the inverse viscosity. ${ }^{12}$ In this work we discuss how particle interactions affect the applicability of generalized SED relations.

\section{BACKGROUND}

\section{A. Short-time rotational diffusion in colloidal sphere dispersions}

For a free Brownian tracer sphere in solvent the rotational diffusion coefficient $D_{0}^{r}$ is time independent and given by Eq. (1). However, when the tracer is dispersed in a colloidal host fluid a separation of time scales occurs. The interaction time scale

$$
\tau_{0}^{I}=\frac{1}{D_{0}^{r}}
$$

separates the rotational dynamics into a short-time $\left(D_{s}^{r}\right)$ and long-time $\left(D_{L}^{r}\right)$ diffusion process. ${ }^{25}$ Equation (3) gives the time roughly needed for a significant change of direct interactions (DI) due to configurational relaxation. At short times the tracer particle rotates in an unchanging equilibrium configuration of neighbor particles, so its short-time diffusion coefficient $D_{s}^{r}$ is a hydrodynamic quantity. ${ }^{2}$ At long times the tracer experiences many independent realizations of the host-particle configuration so diffusion is not only affected by hydrodynamic interactions (HI) but also by DI, i.e., memory effects. As a result, $D_{L}^{r}<D_{s}^{r} .{ }^{26-28}$ Below we summarize only theory for short-time rotational diffusion, 2,12,29-32 which is the relevant regime in our experiments. ${ }^{12}$ Though $D_{s}^{r}$ is not affected directly by DI, they are still important in this regime as they determine the host particle configuration. The theory, summarized below, predicts that in particular the details of the interaction potential near contact have a strong effect on $D_{s}^{r}$.

The quantity of interest is the hydrodynamic function $H_{s}^{r}(\phi)=D_{s}^{r}(\phi) / D_{0}^{r}$ that depends on the host particle volume fraction $\phi$. Due to the many-body nature of HI, $H_{s}^{r}(\phi)$ cannot be evaluated exactly and is therefore approximated by a series expansion in $\phi$,

$$
H_{s}^{r}(\phi) \approx 1+H_{s 1}^{r}(\phi) \phi+H_{s 2}^{r}(\phi) \phi^{2}+\ldots,
$$

which is truncated after the third term. ${ }^{2}$ The coefficient $H_{s 1}^{r}$ represents the configuration-averaged effect on rotational diffusion of two-body $\mathrm{HI}$ between one tracer and one host particle,

$$
H_{s 1}^{r}(\phi)=\frac{1}{D_{0}^{r}} \int_{a_{H}+a_{T}}^{\infty} d r r^{2} g_{\mathrm{TH}}^{(2)}(r, \phi) A_{\mathrm{TH}}^{r r}(r) .
$$

In the following we assume that the tracer radius $a_{T}$ equals the host radius $a_{H}$. Equation (5) integrates pair HI over the equilibrium tracer-host pair correlation function $g_{\mathrm{TH}}^{(2)}(r, \phi)$. The two-body mobility function $A_{\mathrm{TH}}^{r r}(r)$, which can be expressed as a series expansion in even powers of the inverse tracer-host distance $r^{-1}$, relates the angular velocity of a tracer to the torque that it exerts on the fluid. It is very shortranged: the leading term in the series expansion is proportional to $r^{-6.29,30}$ Thus, the main contribution to the integral in Eq. (5) comes from particle configurations near contact. The second coefficient, $H_{s 2}^{r}(\phi, \lambda)$ in Eq. (4) relates to threebody HI between one tracer and two host particles, weighted by the triplet distribution function $g_{\mathrm{THH}}^{(3)} \cdot{ }^{12,32}$

To evaluate $H_{s 1}^{r}$ and $H_{s 2}^{r}$ one needs to specify the pair potential between tracer and host particles, which determines the radial distribution functions $g_{\mathrm{TH}}^{(2)}, g_{\mathrm{HH}}^{(2)}$, and $g_{\mathrm{THH}}^{(3)}$. For uncharged tracer and host spheres it is reasonable to use virial expansions of the distribution functions to first order for $g_{\mathrm{TH}}^{(2)}$ and zeroth order for $g_{\mathrm{THH}}^{(3)}$. However, since $g_{\mathrm{TH}}^{(2)}$ has its maximum at contact, it is necessary to include many terms in the expansion of the mobility tensors in powers of $r^{-1}$. Including lubrication interactions and expanding the two-body mobility to order $r^{-1000}$ and the three-body mobility to $r^{-21}$ leads to ${ }^{30}$

$$
H_{s}^{r} \approx 1-0.6310 \phi-0.726 \phi^{2} .
$$

This equation describes experimental ${ }^{2,7}$ and simulation ${ }^{33}$ results well up to $\phi \approx 0.35$. We note that the near-contact region $2 a_{T} \leqslant r \leqslant 2.1 a_{T}$ contributes $25 \%$ to the first virial coefficient, ${ }^{29}$ indicating the sensitivity of $H_{s}^{r}$ to the region of close approach.

For a dispersion of spheres with surface charge $Z$ the radial distribution functions depend on the amount of electrolyte, which sets the Debye screening length $\kappa^{-1}$,

$$
\kappa^{-1}=\sqrt{\frac{\varepsilon_{0} \varepsilon k_{B} T}{e^{2}\left[n|Z|+2 n_{s}\right]}},
$$

with $n$ the number concentration of colloids and $n|Z|$ the number concentration of (monovalent) counterions, $n_{s}$ the number concentration of added (1-1) electrolyte, $e$ the elementary charge, $\varepsilon$ the solvent dielectric constant, and $\varepsilon_{0}$ the vacuum permittivity. ${ }^{25}$ In the case of incomplete electrolyte dissociation, ${ }^{34}$ the salt concentration $n_{s}$ equals the total concentration of dissolved salt times the dissociation constant $\alpha$, which can be estimated from conductivity measurements (see the Appendix). The screening length $\kappa^{-1}$ determines the range of the repulsive pair potential $V(r)$ between two charged spheres with radius $a$. The functional form of $V(r)$ depends on the boundary conditions; two well-known limiting cases are a double-layer interaction at constant surface potential and at constant surface charge. ${ }^{35}$ Calculations of $H_{s}^{r}(\phi)$ have so far been performed only for the case of constant charge $Z$, using ${ }^{12,25}$

$$
V(r)=\frac{(Z e)^{2}}{\varepsilon_{0} \varepsilon}\left(\frac{\exp (\kappa a)}{1+\kappa a}\right)^{2} \frac{\exp (-\kappa r)}{r} .
$$




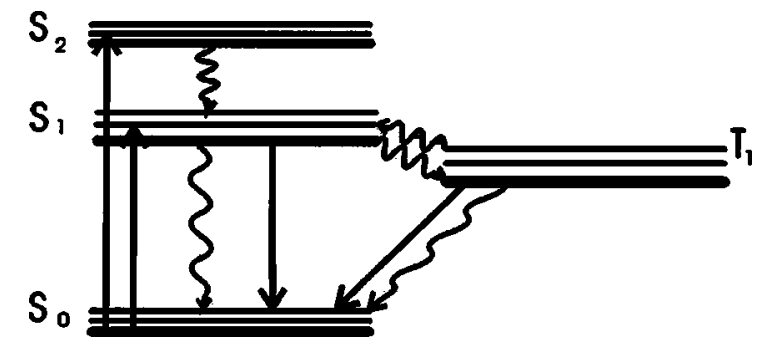

FIG. 1. Jablonski diagram, schematically showing the energy levels involved in fluorescence $\left(S_{1} \rightarrow S_{0}\right)$ and phosphorescence $\left(T_{1} \rightarrow S_{0}\right)$.

Since charged colloids are strongly correlated, the radial distribution functions are no longer given by simple viral expansions, but should be obtained instead from integral equation schemes. ${ }^{25}$ On the other hand, the calculation of $\mathrm{HI}$ simplifies, because the radial distribution functions remain essentially zero for particle separations $r \leqslant \kappa^{-1}$. Since the hydrodynamic mobility functions decay rapidly with $r$, hydrodynamic coupling between two charged spheres is weaker than for two uncharged ones, for which $g_{\mathrm{TH}}^{(2)}$ has a maximum at contact $\left(r=a_{H}+a_{T}\right)$. Thus, $H_{s}^{r}$ increases with decreasing ionic strength or increasing surface charge. ${ }^{25}$ For fully deionized suspensions of highly charged spheres, $H_{s}^{r}$ assumes a quadratic dependence on $\phi$,

$$
H_{s}^{r}=1-a_{r} \phi^{2}
$$

with an essentially charge-independent parameter $a_{r}$ $\approx 1.15^{12,25,31}$

\section{B. Principle of time resolved phosphorescence anisotropy (TPA)}

In a TPA experiment, a sample containing dye-labeled particles is exposed to a brief pulse of vertically polarized light, which excites the phosphorescent dye molecules from the singlet ground state $S_{0}$ to the first excited singlet state $S_{1}$ (see the schematic Jablonski diagram in Fig. 1). ${ }^{11}$ Via intersystem crossing, a certain portion of the excited molecules reverts to the triplet excited state $T_{1}$. Phosphorescence occurs when molecules in the $T_{1}$ state relax to the ground state $S_{0}$ by emitting a photon. In contrast to the $S_{1} \rightarrow S_{0}$ transition (fluorescence), the $T_{1} \rightarrow S_{0}$ transition is spin forbidden. Therefore, the phosphorescence lifetime (typically $10^{-3} \mathrm{~s}$ ) is much longer than the fluorescence lifetime $\left(\sim 10^{-9} \mathrm{~s}\right)$. The lifetime of eosin, used in this paper, is about $3 \mathrm{~ms}^{11}$

The probability for absorption of a photon by a dye molecule with absorption dipole moment $\boldsymbol{\mu}_{\mathrm{a}}$ is proportional to the square of the inner product between $\boldsymbol{\mu}_{\mathrm{a}}$ and the polarization direction of the excitation pulse $\mathbf{E}$. This implies that a polarized light pulse produces an orientationally anisotropic subset of excited-state dye molecules. If the molecule's emission dipole moment $\boldsymbol{\mu}_{\mathrm{e}}$ is parallel to $\boldsymbol{\mu}_{\mathrm{a}}$, then immediately after excitation the intensity of vertically $\left(I_{V V}\right)$ polarized phosphorescence is higher than the horizontally polarized component $\left(I_{V H}\right)$. In time the sphere orientations randomize, leading to orientational randomization of the original subset of excited state molecules. Thus, the difference $I_{V V}-I_{V H}$ vanishes. Simultaneously, the total phosphorescent intensity
TABLE I. Densities $\rho_{0}$, shear viscosities $\eta_{0}$, refractive indices $n_{0}$, and permittivities $\varepsilon$ of pure solvents and their mixtures. The superscripts denote temperature in ${ }^{\circ} \mathrm{C}$.

\begin{tabular}{llccc}
\hline \hline \multicolumn{1}{c}{ Solvent } & $\eta_{0}(\mathrm{mPa} \mathrm{s})^{\mathrm{a}}$ & $\rho_{0}(\mathrm{~g} / \mathrm{mL})^{\mathrm{b}}$ & $n_{0}$ & $\varepsilon$ \\
\hline DMF & $0.795054^{25}$ & $0.94451^{25}$ & $1.430^{20, \mathrm{c}}$ & $37.6^{23, \mathrm{c}}$ \\
DMSO & $1.940899^{25}$ & $1.09539^{25}$ & $1.476^{20, \mathrm{c}}$ & $47.1^{23, \mathrm{c}}$ \\
DMF-DMSO & $1.368024^{23}$ & $1.03731^{23}$ & $1.4589^{20, \mathrm{~d}}$ & $43.3^{23, \mathrm{~d}}$ \\
& $1.313318^{25}$ & $1.03534^{25}$ & & \\
Ethanol & $1.106819^{23}$ & $0.78729^{23}$ & $1.3611^{20, \mathrm{c}}$ & $25.1^{23, \mathrm{c}}$ \\
& $1.087007^{25}$ & & & \\
Toluene & $0.562^{23}$ & $0.86395^{23}$ & $1.4961^{20, \mathrm{c}}$ & $2.38^{23, \mathrm{c}}$ \\
Ethanol-toluene & $0.63732^{23}$ & $0.84156^{23}$ & $1.4578^{25, \mathrm{~d}}$ & $9.19^{23, \mathrm{~d}}$ \\
& $0.618956^{25}$ & $0.83964^{25}$ & & \\
\hline \hline
\end{tabular}

${ }^{\mathrm{a}}$ Measured with an Ubbelohde capillary viscometer.

${ }^{\mathrm{b}}$ Measured with an Anton-Paar densitometer.

${ }^{c}$ Literature values (Ref. 77).

${ }^{\mathrm{d} C a l c u l a t e d}$ from values for pure solvents assuming a linear dependence on the solvent composition in terms of volume fractions.

$$
I_{p}(t)=I_{V V}(t)+2 I_{V H}(t)
$$

decays to zero because the excited triplet state $T_{1}$ relaxes to the singlet ground state $S_{0} \cdot{ }^{11}$ In the case of eosin this process imposes an upper limit of $\sim 10 \mathrm{~ms}$ to the experimentally accessible rotational correlation times.

The rotational diffusion coefficient can be obtained from the anisotropy $r_{p}(t)$, defined as

$$
r_{p}(t)=\frac{I_{V V}(t)-I_{V H}(t)}{I_{p}(t)} .
$$

Since $r_{p}(t)$ is normalized by $I_{p}(t)$, its time dependence is completely determined by rotational diffusion of the particle carrying immobilized dye molecules. The anisotropy gives the ensemble (or time) averaged correlation between the absorption dipole moment $\boldsymbol{\mu}_{\mathrm{a}}(0)$ at time $t=0$, and the emission dipole moment $\boldsymbol{\mu}_{\mathbf{e}}(t)$ at time $t:^{36}$

$$
r_{p}(t)=\frac{2}{5}\left\langle P_{2}\left[\mu_{a}(0) \mu_{e}(t)\right]\right\rangle,
$$

with $P_{2}$ the second Legendre polynomial. The functional form of $\left\langle P_{2}\right\rangle$ depends on the nature of the reorientation process. ${ }^{37,38}$ For the simplest case of isotropic Brownian motion of noninteracting monodisperse tracer spheres ${ }^{37}$

$$
r_{p}(t)=r_{p}(0) e^{-6 D^{r} t}
$$

with $D^{r}$ the rotational diffusion coefficient. The value of the zero-time anisotropy $r_{p}(0)$ has a theoretical maximum of 0.4. However, in the case of eosin the maximum zero-time anisotropy is reduced to 0.19 , because the phosphorescence emission dipole moment $\boldsymbol{\mu}_{\mathbf{e}}$ makes an angle of $36^{\circ}$ with the excitation dipole moment $\boldsymbol{\mu}_{\mathbf{a}} \cdot{ }^{39}$ In practice $r_{p}(0)$ is usually further lowered due to energy transfer between dye molecules and rotation of the dye molecules (i.e., the emission dipole moment) within the free volume accessible to them inside the colloidal particles. 
TABLE II. Debye screening length $\kappa^{-1}$, mass density $\rho_{0}$, and shear viscosity $\eta_{0}$ for the solvent mixtures DMSO-DMF and toluene-ethanol, for various $\mathrm{LiCl}$ and $\mathrm{LiNO}_{3}$ concentrations, respectively.

\begin{tabular}{llcllll}
\hline \hline Solvent & Property & $0.1 \mathrm{mM}$ & $1 \mathrm{mM}$ & $10 \mathrm{mM}$ & $100 \mathrm{mM}$ & $500 \mathrm{mM}$ \\
\hline DMSO-DMF & $\kappa^{-1}(\mathrm{~nm})^{\mathrm{a}}$ & 22.3 & 7.1 & 2.3 & 0.74 & 0.33 \\
& $\rho_{0}(\mathrm{~g} / \mathrm{mL})^{\mathrm{b}}$ & 1.03534 & 1.03535 & 1.03546 & 1.03872 & 1.05191 \\
& $\eta_{0}(\mathrm{mPa} \mathrm{s})^{\mathrm{c}}$ & 1.31332 & 1.31526 & 1.33295 & 1.43177 & 1.95057 \\
\multirow{2}{*}{ Tol-EtOH } & & & & & \\
& $\kappa^{-1}(\mathrm{~nm})^{\mathrm{a}}$ & 12.1 & 6.0 & 2.8 & 0.88 & - \\
& $\rho_{0}(\mathrm{~g} / \mathrm{mL})^{\mathrm{b}}$ & 0.83964 & 0.83970 & 0.84028 & 0.84503 & - \\
& $\eta_{0}(\mathrm{mPa} \mathrm{s})^{\mathrm{c}}$ & 0.61896 & 0.61950 & 0.62431 & 0.68058 & - \\
\hline \hline
\end{tabular}

${ }^{a}$ Calculated using Eq. (7), accounting for incomplete electrolyte dissociation as explained in the Appendix.

${ }^{\mathrm{b}}$ Measured with an Anton-Paar densitometer.

${ }^{c}$ Measured with an Ubbelohde capillary viscometer.

\section{EXPERIMENTAL METHODS}

\section{A. Colloid synthesis and characterization}

\section{Solvents}

Two refractive-index matching solvent mixtures were used, namely $3: 2 \mathrm{v} / \mathrm{v}$ dimethylsulfoxide-N,N-dimethylformamide (DMSO-DMF) for uncoated silica spheres, and $7: 3 \mathrm{v} / \mathrm{v}$ toluene-ethanol for silica spheres coated with the silane coupling agent 3-methacryloxy propyltrimethoxysilane (TPM). In both cases the silica particles acquire a negative surface charge due to dissociation of the acid silanol groups on the silica surface (and those of the TPM oligomers). ${ }^{40}$ Table I shows some physical properties of the solvents and their mixtures.

The Debye screening length $\kappa^{-1}$ was controlled by adding variable amounts of electrolyte ( $\mathrm{LiCl}$ in DMSO-DMF and $\mathrm{LiNO}_{3}$ in toluene-ethanol). Values of $\kappa^{-1}$ (Table II) were calculated using Eq. (7) with the salt concentration $n_{s}$ corrected for incomplete electrolyte dissociation using results from conductivity measurements (see Appendix). An upper limit to the counterion concentration (assuming univalent ions) in the solvent can be estimated as follows:

$$
\begin{aligned}
c(\mathrm{~mol} / \mathrm{L}) & \leqslant\left(\frac{1}{1-\phi_{\max }}\right) \frac{1}{1000 N_{\mathrm{av}}} n_{\max }|Z| \\
& =\left(\frac{1}{1-\phi_{\max }}\right) \frac{3 \phi_{\max }}{4 \pi a_{H}^{3}} \frac{|Z|}{1000 N_{\mathrm{av}}},
\end{aligned}
$$

where $N_{\text {av }}$ is Avogadro's number and $\phi_{\max }$ represents the highest volume fraction studied $\left(\phi_{\max }=0.35\right.$ in DMSO-
DMF). Using $Z=220$, which earlier gave good agreement between experimental and theoretical values of $H_{s}^{r}$ for our silica particles, ${ }^{12} \mathrm{Eq}$. (14) yields $c \leqslant 0.1 \mathrm{mM}$. This corresponds to a screening length $\kappa^{-1} \geqslant 22 \mathrm{~nm}$ in DMSO-DMF with $c_{\mathrm{LiCl}}=0$. Measurements of the host dispersion lowshear viscosity $\eta_{L}(\phi)$ demonstrated that the residual ionic strength was certainly less than $1 \mathrm{mM} \mathrm{LiCl}$, since adding 1 $\mathrm{mM} \mathrm{LiCl}$ to an initially "salt-free" suspension led to a significant decrease of $\eta_{L}(\phi) / \eta_{L}(0)$ from 68 to 11 at $\phi$ $=30 \%$ (result not shown). For TPM-silica in tolueneethanol we estimate a similar charge number as for bare silica spheres in DMSO-DMF, giving $\kappa^{-1} \geqslant 12 \mathrm{~nm}$ for $c_{\mathrm{LiNO}_{3}}=0$. Table II also lists the mass density $\rho_{0}$ and viscosity $\eta_{0}$ of the solvent mixtures for the $\mathrm{LiCl}$ concentrations used.

\section{Tracer and host spheres in DMSO-DMF}

Tracer silica spheres coded "eR75" were prepared using the familiar Stöber recipe, ${ }^{41}$ and labeled with eosin-5isothiocyanate (EoITC) applying a procedure originally developed for fluorescein-ITC. ${ }^{42}$ The particles, with a final radius of $96 \mathrm{~nm}$ (see Table III), consisted of a phosphorescent silica core of $40 \mathrm{~nm}$ covered with a nonphosphorescent silica shell. Tracer silica spheres coded " $\mu 10$ " and " $\mu 30$ " were prepared by coating nonphosphorescent cores obtained by a microemulsion pathway ${ }^{43}$ (radius $22 \mathrm{~nm}$ ) with a $19 \mathrm{~nm}$ thick phosphorescent Stöber silica shell (giving particles coded $\mu 0$ ), followed by one (in the case of $\mu 10$ ) or two (in the case of $\mu 30)$ nonphosphorescent silica shells. Note that tracers $\mu 10$ and $\mu 30$ have a significantly smaller polydispersity than

TABLE III. Particle radii $a_{\mathrm{DLS}}$ and $a_{\mathrm{TPA}}$, relative size polydispersity $\sigma_{\mathrm{TEM}}$, number of dye molecules per tracer particle $n_{\text {dye }}$, label efficiency $e \%$, initial phosphorescence anisotropy $r_{p}(0)$, and rotational decay time $\tau_{\text {rot }}$.

\begin{tabular}{llcccccc}
\hline \hline \multicolumn{1}{c}{ Particle } & $\sigma_{\text {TEM }^{\mathrm{a}}}$ & $\begin{array}{c}a_{\mathrm{DLS}}{ }^{\mathrm{b}} \\
(\mathrm{nm})\end{array}$ & $\begin{array}{c}a_{\mathrm{TPA}}{ }^{\mathrm{c}} \\
(\mathrm{nm})\end{array}$ & $n_{\text {dye }}$ & $e \%$ & $r_{p}(0)^{\mathrm{c}}$ & $\begin{array}{c}\tau_{\text {rot }}{ }^{\mathrm{c}} \\
(\mathrm{ms})\end{array}$ \\
\hline$\mu 0$ & - & 41 & - & 225 & 27 & - & - \\
$\mu 10$ & $1.5 \%$ & 72 & 67.2 & 1345 & 70 & 0.108 & 0.39 \\
$\mu 30$ & $2.7 \%$ & 100 & 102.5 & 1439 & 77 & 0.110 & 1.44 \\
eR75 & $9 \%$ & 99 & 96.3 & 315 & 31 & 0.138 & 1.19 \\
eR75-TPM & $8 \%$ & 85 & 90.9 & 315 & 31 & 0.138 & 0.47 \\
R75 & $8 \%$ & 95.0 & - & 0 & - & - & - \\
R75-TPM & $8 \%$ & 90.3 & - & 0 & - & - & - \\
\hline \hline
\end{tabular}

${ }^{\mathrm{a}}$ Determined from electron micrographs.

${ }^{\mathrm{b}}$ Measured with dynamic light scattering (DLS).

${ }^{\mathrm{c}}$ Measured with TPA. 
tracer eR75 (Table III). Host silica spheres labeled R75 with equal size and polydispersity (cf. Table III) as the eR75 tracers were prepared according to the same Stöber procedure. ${ }^{41}$ All tracer and host particles were transferred to $3: 2 \mathrm{v} / \mathrm{v}$ DMSO-DMF by centrifugation.

\section{Tracer and host particles in toluene-ethanol}

Silica spheres flocculate in the presence of toluene, but when coated with a layer $(2-4 \mathrm{~nm})$ of TPM they are stable in toluene-ethanol mixtures. ${ }^{40}$ Aggregation of TPM-silica only occurs if a large excess of toluene is present. This system is therefore suitable for tuning particle attractions by changing the solvent composition. A portion of the eR75 tracer and R75 host particles was coated with a covalently linked TPM layer following Ref. 40. The particles were transferred to 7:3 v/v toluene-ethanol by repeated centrifugation. Characterization results are shown in Table III.

\section{Particle characterization}

The concentration of eosin inside the tracer particles was determined by optical absorption measurements after dissolution of the particles in $1 \mathrm{M} \mathrm{NaOH}$ in water. Absorption spectra were measured between 350 and $650 \mathrm{~nm}$ and corrected for baseline and scattering by subtracting a spectrum of dissolved undyed particles (radius $39 \mathrm{~nm}$ ). The area under the peak (centered around $520 \mathrm{~nm}$ ) was taken as a measure of eosin concentration. Absolute concentrations were obtained by comparing with a calibration series of EoITC $(1-10 \mu \mathrm{M})$ in $1: 1.5: 15 \mathrm{DMF} / \mathrm{DMSO} / \mathrm{NaOH}(1 \mathrm{M})$. The number of dye molecules $n_{\text {dye }}$ per particle is shown in Table III. The labeling efficiency e $\%$ of both particles $\mu 0$ and eR75 is about $30 \%$, in agreement with previous observations on EoITC labeled Stöber silica. ${ }^{11}$ The labeling efficiency of $\mu 10$ and $\mu 30$ is higher than for $\mu 0$, indicating that the second and third "nonphosphorescent" silica shells actually also contain eosin. Apparently, unreacted EoITC that remains after formation of $\mu 0$ is incorporated during the subsequent growth steps.

Particle volume fractions were determined by the weight loss on drying using particle densities of $1.73 \mathrm{~g} / \mathrm{cm}^{3}$ for bare silica and $1.46 \mathrm{~g} / \mathrm{cm}^{3}$ for TPM-silica. Number-averaged particle size polydispersities $\sigma$ were determined from transmission electron micrographs (TEM). Hydrodynamic radii $a_{\text {DLS }}$ and $a_{\mathrm{TPA}}$ were derived from $D_{0}^{t}$ measured with dynamic light scattering (DLS) and $D_{0}^{r}$ measured with TPA, using Eqs. (1) and (2). There was good agreement between $a_{\mathrm{DLS}}$ and $a_{\mathrm{TPA}}$ (Table III). For bare R75 particles, $a_{\text {DLS }}$ depended on the solvent composition: $a_{\mathrm{DLS}}=95 \mathrm{~nm}$ in DMF, DMSO, and DMSO-DMF, while $a_{\text {DLS }}=90 \mathrm{~nm}$ in ethanol. This difference, previously observed also by Imhof et al. ${ }^{23}$ is very likely due to strong solvation of silica by DMF and DMSO (cf. Sec. IV A). In DMSO-DMF, $a_{\text {DLS }}$ was independent of $c_{\mathrm{LiCl}}$ over the whole range studied $(0-500 \mathrm{mM})$, indicating that electrolyte friction effects are negligible. For TPM coated R75 particles the DLS radius in ethanol $(90.5 \mathrm{~nm})$ was equal to that in toluene-ethanol $(90.3 \mathrm{~nm})$. (The particles were unstable in pure toluene.)

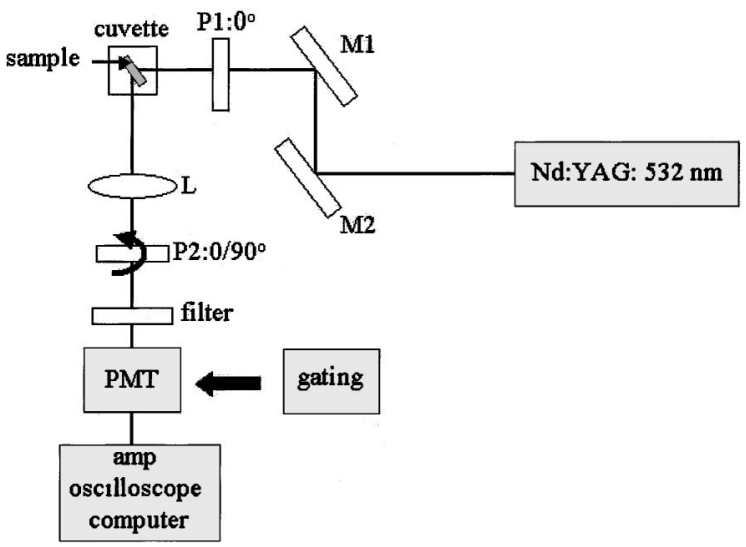

FIG. 2. Schematic depiction of TPA setup (not drawn to scale). The Nd:YAG laser provides $5 \mathrm{~ns}$ light pulses. Via two mirrors (M1 and M2) and a Glan-Tayler polarizer $\left(\mathrm{P} 1\right.$, orientation $\left.0^{\circ}\right)$ the light is directed onto the sample, contained in a glass capillary placed in a cuvette filled with toluene. Phosphorescent light is detected under an angle of $90^{\circ}$ with respect to the incoming beam. The vertical and horizontal components are measured alternately by switching a sheet polarizer (P2) placed in front of the PMT between $0^{\circ}$ and $90^{\circ}$. Scattered light is discarded by an interference filter.

\section{B. Rheology and TPA}

Low-shear viscosity measurements were performed using a Contraves Low Shear 40 rheometer thermostatted at $23.0^{\circ} \mathrm{C}$. Viscosities $\eta_{L}$ were determined from the slope of shear stress versus shear rate (between 0 and $50 \mathrm{~s}^{-1}$ ) plots.

TPA samples were typically prepared by addition of 5 $\mu \mathrm{L}$ concentrated tracer stock dispersion to $100-150 \mu \mathrm{L}$ of host dispersion. The final tracer volume fraction was $0.4 \%-$ $1 \%$. The TPA measurements were performed at $23^{\circ} \mathrm{C}$, using a modified version of the setup described by Lettinga $e t$ al. ${ }^{44}$ (see Fig. 2). The eosin dye was excited by short (5 ns) pulses from a Nd:yttrium-aluminum-garnet (YAG) laser (Continuum MiniLite II) with a wavelength of $532 \mathrm{~nm}$. The vertical $(V)$ polarization of the incident beam was improved with a Glan-Tayler prism. The samples were contained in narrow glass capillaries immersed in toluene to optically match the glass, and kept at an angle of $45^{\circ}$ to the incident light beam. Phosphorescent light was detected at an angle of $90^{\circ}$ relative to the incident beam, using an electronically gated photomultiplier (PMT), amplifier (Melles-Griot), and oscilloscope (LeCroy). Measurements consisted of several sequences of alternately measuring horizontal $\left(I_{V H}\right)$ and vertical $\left(I_{V V}\right)$ polarized emission. The polarization direction was selected with a sheet polarizer placed in front of the PMT. The intensities were corrected for the polarization sensitivity of the detection line $G=I_{H H} / I_{H V}=0.86$. Anisotropy decay curves $r_{p}(t)$ were well described by Eq. (13), except where explicitly mentioned in the text.

\section{RESULTS AND DISCUSSION}

\section{A. Silica sphere suspensions in DMSO-DMF}

\section{Colloidal stability of silica in DMSO-DMF}

The silica sols in 3:2 v/v DMSO-DMF showed remarkable stability toward flocculation by electrolyte up to $\mathrm{LiCl}$ concentrations of $c_{\mathrm{LiCl}}=400 \mathrm{mM}$. No signs of flocculation 
were observed over a period of at least 2 years van der Waals attractions between silica spheres in DMSO-DMF are small due to close refractive index matching and in addition screened by strong solvent adsorption, as suggested by the 5 $\mathrm{nm}$ larger hydrodynamic radius of the particles in DMSODMF as compared to ethanol (cf. Sec. III A 4). A similar difference in hydrodynamic radii $(3 \mathrm{~nm})$ was reported by Imhof et al. ${ }^{23}$ between silica in DMF and in ethanol. ${ }^{23} \mathrm{Re}-$ cently, significant adsorption enthalpies have been measured for silica in DMF and in DMSO $\left(\sim 0.2 \mathrm{~J} / \mathrm{m}^{2}\right.$ in both cases, compared to $0.07 \mathrm{~J} / \mathrm{m}^{2}$ for undecane $)^{45}$ using an immersion experiment. ${ }^{46}$ The adsorption enthalpies of DMF and DMSO are similar to that of water, which is known to strongly hydrate colloidal silica. Direct force measurements between silica surfaces in water ${ }^{47}$ have shown that hydration leads to a strong monotonic repulsion for surface separations between 1 and $5 \mathrm{~nm}$. The exceptional stability of silica sols in water $^{48,49}$ at high ionic strength, even close to the isoelectric point, is probably related to this repulsion. The repulsion has been variously attributed to the presence of a structured layer of water at the silica surface ${ }^{47}$ or to formation of a gel layer at the silica surface in the presence of water. This gel layer could be a water-swollen diffuse silica layer with a Hamaker constant almost equal to that of water ${ }^{50}$ or a layer with protruding silanol and silicic acid groups that afford steric stabilization. $^{49,51}$ It is plausible that DMF and DMSO give rise to a similar solvation force. Since intermolecular hydrogen bonding is negligible in DMF and DMSO, we suggest that it is unlikely that a modified solvent structure near the surface can account for a 3-5 $\mathrm{nm}$ increase in hydrodynamic radius ( $\sim 10-15$ solvent layers). Instead, it seems likely that DMF and DMSO dissolve, or diffuse into and swell, the silica surface and form a gel layer. Colloidal stability in DMSO-DMF was lost only when $500 \mathrm{mM} \mathrm{LiCl}$ was added. However, even then particle aggregation was slow (see below).

\section{TPA measurements of short-time rotational tracer diffusion}

Figure 3(A) shows the reduced rotational diffusion coefficient $H_{s}^{r}$ of eR75 tracer spheres in suspensions of R75 host spheres in DMSO-DMF, as a function of host particle volume fraction $\phi$ for various concentrations of added $\mathrm{LiCl}$, $c_{\mathrm{LiCl}}$. At all ionic strengths investigated rotational diffusion monotonically slows down with $\phi$. The $\phi$ dependences are fairly well described by second-order virial expressions (dotted lines). The drawn and dashed lines represent Eq. (6) for $H_{s}^{r}$ of hard-sphere suspensions and Eq. (9) for $H_{s}^{r}$ of fully de-ionized suspensions of charged spheres. Diffusion of charged spheres is faster than that of hard spheres due to weaker hydrodynamic coupling. In the range $c_{\mathrm{LiCl}}$ $=0-100 \mathrm{mM}$ the data lie between the two theoretical predictions, while at $c_{\mathrm{LiCl}}=500 \mathrm{mM}$ the data fall below the hard-sphere prediction.

At the lowest ionic strength (zero added $\mathrm{LiCl}$ ) rotational diffusion is slower than predicted by Eq. (9) and follows a linear rather than a quadratic $\phi$ dependence at small $\phi$. This is most likely due to the presence of residual electrolyte $(\sim 0.1 \mathrm{mM})$. When more than $10 \mathrm{mM} \mathrm{LiCl}$ was added, rota-
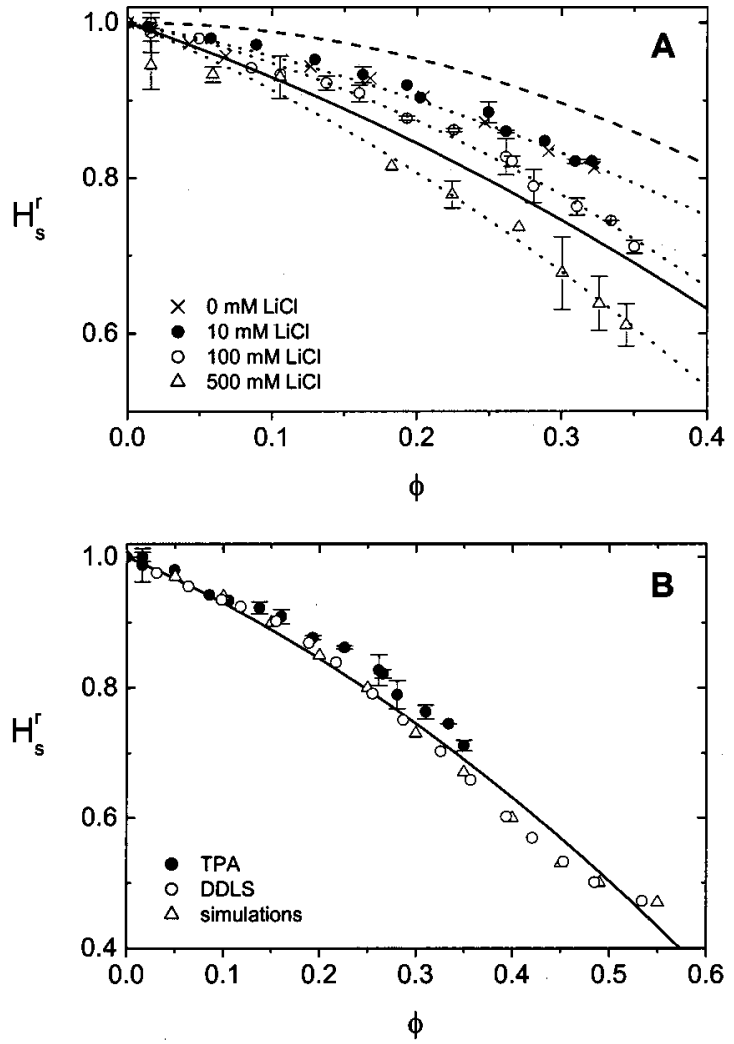

FIG. 3. (A) Reduced short-time rotational tracer diffusion coefficient $H_{s}^{r}$ of eR75 tracers dispersed in R75 host suspensions in 3:2 v/v DMSO-DMF for added $\mathrm{LiCl}$ concentrations as indicated. The dotted lines are second-order polynomial fits; the dashed and solid lines are theoretical expressions Eq. (9) for fully de-ionized dispersions of charged spheres and Eq. (6) for hardsphere suspensions. (B) TPA data in the hard-sphere limit (100 mM LiCl), compared with Eq. (6) (solid line), DDLS data (Ref. 2), and simulation results (Ref. 33).

tional diffusion became slower, in agreement with the theory summarized in Sec. II B. With increased electrostatic screening, hydrodynamic coupling becomes stronger so $H_{s}^{r}$ decreases. However, the data for $0 \mathrm{M}\left(\kappa a_{T}=38\right)$ and $10 \mathrm{mM}$ $\mathrm{LiCl}\left(\kappa a_{T}=4\right)$ coincide within experimental error, even though addition of $10 \mathrm{mM} \mathrm{LiCl}$ did reduce the low-shear viscosity dramatically (see below). This observation is at variance with theoretical calculations using input parameters corresponding to our experimental model system, ${ }^{12}$ which predict a significant difference between $H_{s}^{r}$ at 0 and $10 \mathrm{mM}$ $\mathrm{LiCl}$. Apparently, the theory does not incorporate a complete description of the dispersion. One feature not present in the theory is the strong solvation of silica in DMSO-DMF, which causes a short-range repulsion. Another feature not yet incorporated in the theory is the nature of the charge regulating mechanism, which becomes important when the particles are close. In the calculations the charge $Z$ was fixed (to 220), while in reality spheres near contact may discharge each other to some extent. (The boundary condition of a constant surface potential would necessitate such a discharge.) Interestingly, TPA results for eR75 diffusion in dispersions of larger host spheres $\left(\mathrm{SC}_{07}, a_{T} / a_{H}=0.33\right)$ did show an immediate reduction of $H_{s}^{r}$ on addition of $10 \mathrm{mM}$ $\mathrm{LiCl}$, in accordance with theory [Fig. 4(B) in Ref. 12, $Z_{T}$ $=220$ and $\left.Z_{H}=1200\right]$. 

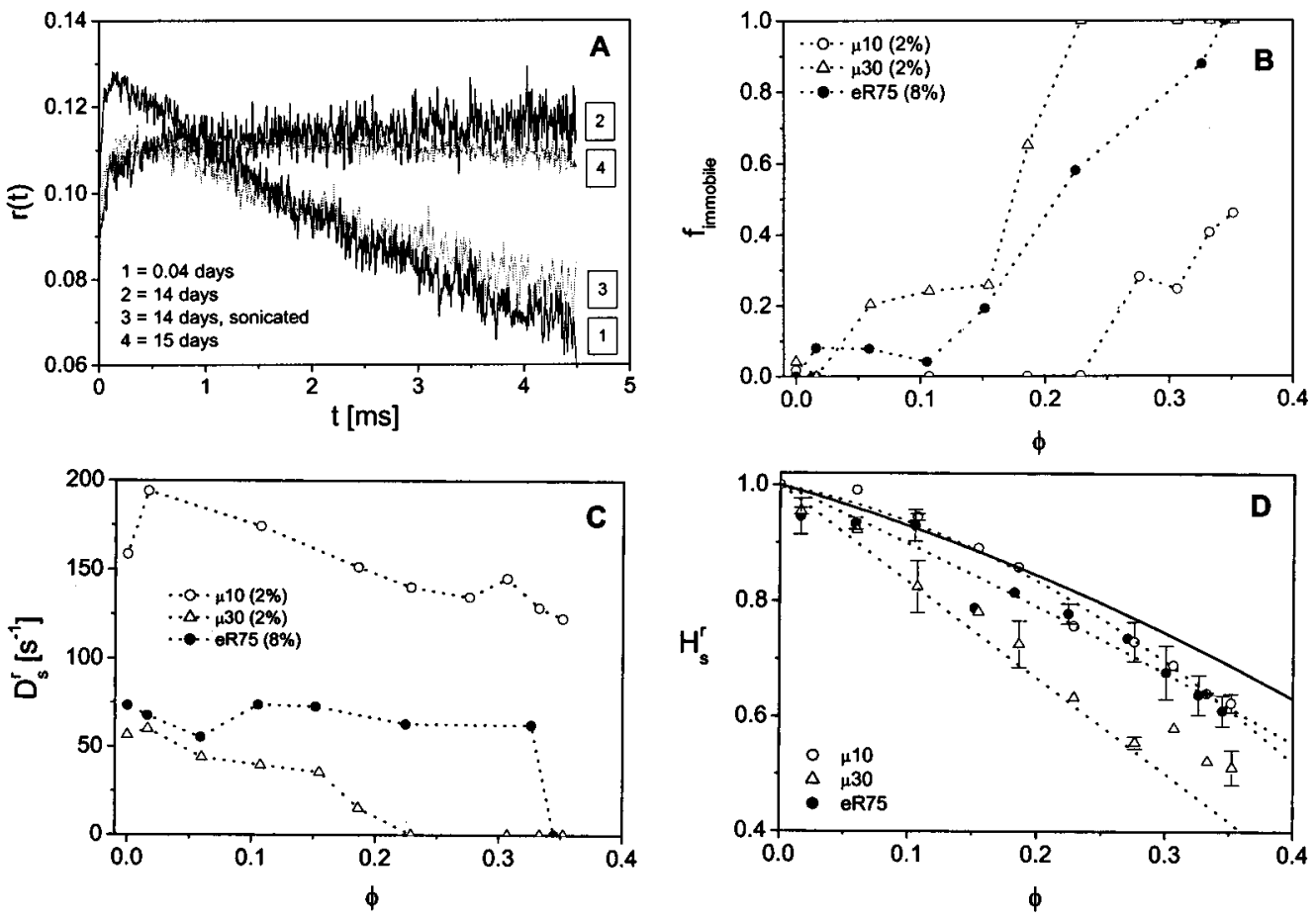

FIG. 4. TPA experiments on suspensions of R75 host spheres in 3:2 v/v DMSO-DMF with $500 \mathrm{mM} \mathrm{LiCl}$. (A) Anisotropy $r_{p}(t)$ for eR75 tracers in a R75 suspension with $\phi=0.33$, measured $0.04,14$, and 15 days after sample preparation. (B) Fraction of immobilized eR75, $\mu 10$, and $\mu 30$ tracers, $f_{\text {immobile }}, 14$ days after sample preparation. (C) Rotational diffusion coefficients $D_{s}^{r}(\phi)$ of the mobile fraction of eR75, $\mu 10$, and $\mu 30$ tracers. (D) Reduced rotational diffusion coefficients $H_{s}^{r}$ of tracers eR75, $\mu 10$, and $\mu 30$ in freshly prepared samples. The dotted lines are to guide the eye; the solid line is Eq. $(6)$ for $H_{s}^{r}(\phi)$ of hard spheres.

At $c_{\text {LiCl }}=100 \mathrm{mM}$, the TPA data almost coincide with the hard-sphere prediction Eq. (6). This is more clearly visualized in Fig. 3(B), where the data are replotted together with DDLS measurements for teflon spheres in water with $\kappa a_{T}$ $=1\left(100 \mathrm{mM} \mathrm{NaCl}, \kappa^{-1}=0.96 \mathrm{~nm}\right)^{2,7}$ and hard sphere simulation results. ${ }^{33}$ There is fair agreement between these data sets and with Eq. (6). ${ }^{30}$ In the region $\phi=0.13-0.26$ the TPA diffusion coefficients are somewhat faster than predicted for hard spheres. This suggests that apart from the hard-core repulsion there is an additional short-ranged repulsive interaction which prevents the particles from coming into close contact. This could be residual electrostatic repulsion. The DDLS results ${ }^{2}$ for $H_{s}^{r}$ also lie slightly above the hard-sphere prediction at small $\phi$, which was tentatively attributed in Ref. 2 to residual electrostatic repulsions. Alternatively, the short-range repulsion is caused by solvation. Note that $\kappa^{-1}$ at $c_{\mathrm{LiCl}}=100 \mathrm{mM}$ is smaller than the solvation layer thickness. Either way, $H_{s 1}^{r}$ would be reduced because the integral in Eq. (5) weighs mainly configurations of neartouching particles due to the very short range of the hydrodynamic mobility functions. For hard spheres the region $2 a_{T} \leqslant r \leqslant 2.1 a_{T}$ contributes $25 \%$ to the prefactor of the order $\phi$ term in Eq. (6). ${ }^{29}$ Thus, any significant repulsion in this range will greatly reduce $H_{s 1}^{r}$, making rotational diffusion very sensitive to even slight deviations from a hard-sphere interaction.

The data obtained at $c_{\mathrm{LiCl}}=500 \mathrm{mM}$ clearly fall below the theoretical prediction for hard spheres [cf. Fig. 3(A)], suggesting that attractive particle interactions play a role. This was confirmed by the viscosity of the host dispersions, which slowly increased over a period of several days. Also, the viscosity measured within $1 \mathrm{~h}$ after salt addition was higher than predicted for hard spheres (see below). Moreover, TPA experiments on samples several days after their preparation showed that the tracer particles became increasingly immobilized.

Figure 4(A) shows anisotropy decay curves $r_{p}(t)$ for a sample containing $\mu 30$ tracers in a R75 host dispersion with $\phi=0.33$. Shortly after sample preparation (curve 1) the anisotropy on the TPA time scale still decayed from 0.125 to 0.07 , indicating that the sample contained mobile species. However, after two weeks (curve 2) the anisotropy remained constant up to $5 \mathrm{~ms}$, suggesting that the tracer particles were frozen on the TPA time scale. After ultrasonication (curve 3) there was again a mobile fraction of tracer particles, indicating that particle aggregation is to some extent reversible. However, one day after this ultrasonic treatment (curve 4) the tracer particles were again immobilized.

From the anisotropy decay curves one can estimate the relative amounts of mobile and immobile (on the TPA time scale) particles. Mobile particles give an exponential decay of the anisotropy $r_{p}(t)$ as in Eq. (13), while immobile particles give a constant anisotropy $A$. Taken together this gives

$$
r_{p}(t)=A+r_{p}(0) \exp \left(-6 D_{s}^{r} t\right) \text {. }
$$

$D_{s}^{r}$ represents the average rotational diffusion rate of species that are mobile on the TPA time scale. In Fig. 4(B) we show the fraction of immobilized particles $f_{\text {immobile }}=A /\left(r_{p}(0)\right.$ $+A$ ) for three different tracers in R75 host dispersions measured 2 weeks after sample preparation. The larger tracers 

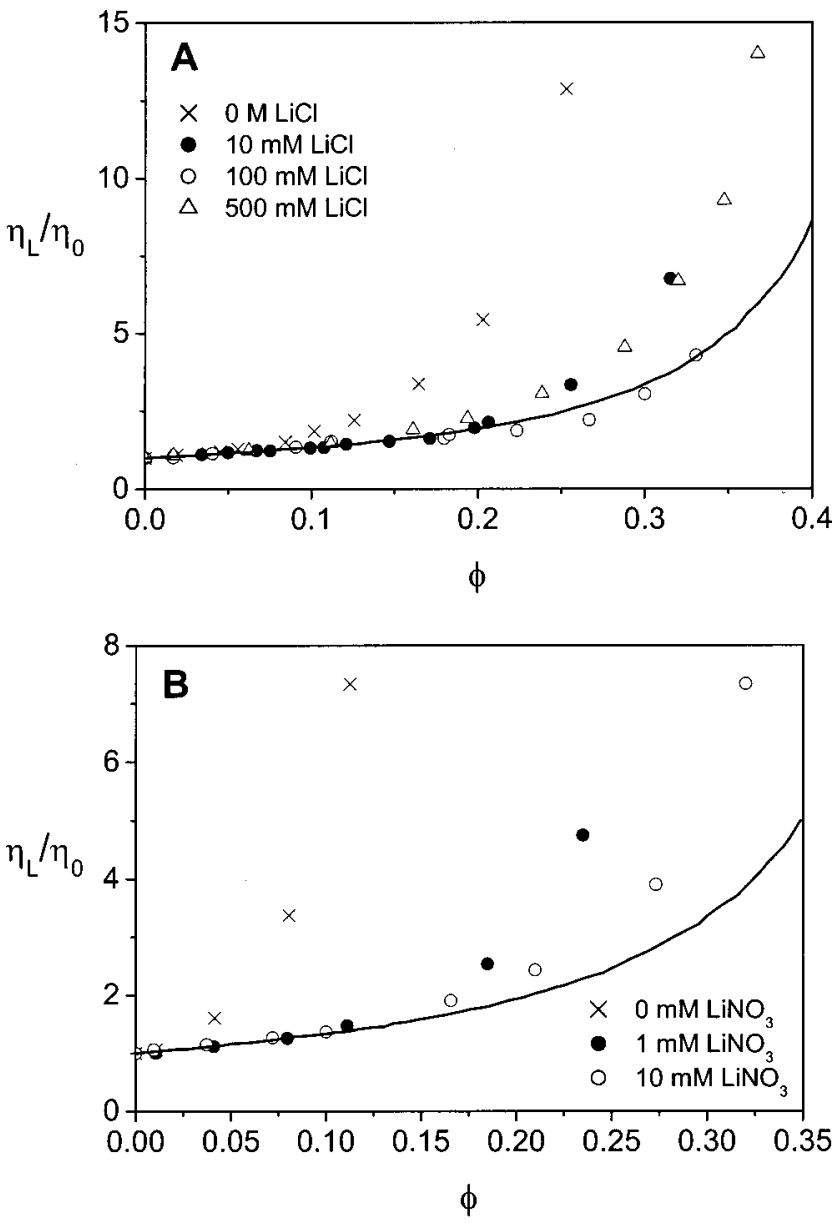

FIG. 5. Reduced low-shear-limiting viscosity $\eta_{L}(\phi) / \eta_{0}$ of host suspensions of (A) R75 spheres in DMSO-DMF (3:2 v/v) with various amounts of $\mathrm{LiCl}$ and (B) R75-TPM spheres in toluene-ethanol $(7: 3 \mathrm{v} / \mathrm{v})$ with various amounts of $\mathrm{LiNO}_{3}$. Drawn lines indicate a mode-coupling theory prediction for hard spheres (Ref. 22).

(eR75 and $\mu 30$ ) were completely immobilized at the highest host particle volume fractions, while the smallest tracer $\mu 10$ remained partially mobile. The eR75 tracers were completely immobilized for $\phi \geqslant 35 \%$, while the $\mu 30$ tracers, which have a similar number-average radius, were immobile already at $\phi=20 \%$. This difference is perhaps due to the larger polydispersity of the eR75 tracers compared to $\mu 30$ (Table III). The mobility of the mobile species remained more or less constant over the whole $\phi$ range [Fig. 4(C)], in contrast to the marked $\phi$ dependence of rotational diffusion observed immediately after sample preparation [Fig. 4(D)]. The reduced diffusion coefficients $H_{s}^{r}$ corresponding to the data in Fig. $4(\mathrm{C})$ are of order 0.4 for both $\mu 10, \mu 30$, and eR75.

\section{Low-shear viscosity of host suspensions}

For comparison with the rotational tracer mobility in host suspensions we also measured the low-shear viscosities $\eta_{L}$ of the host fluids. Figure 5(A) shows the reduced lowshear-limiting viscosity $\eta_{L}(\phi) / \eta_{0}$ of R75 suspensions in DMSO-DMF. At low $c_{\mathrm{LiCl}}$, the measured $\eta_{L}(\phi) / \eta_{0}$ was larger than predicted by mode-coupling theory calculations for hard spheres ${ }^{22}$ (cf. drawn line). With increasing $c_{\mathrm{LiCl}}$, the viscosity decreased until at $c_{\mathrm{LiCl}}=100 \mathrm{mM}$ it corresponded to the hard sphere prediction. This confirms that particle interactions in DMSO-DMF are dominated by long-range electrical double layer repulsions: On addition of salt the repulsion is screened [Eq. (7)] so the viscosity decreases. ${ }^{52-55}$ If attractive interactions would dominate, salt addition would enhance the viscosity. ${ }^{53,54}$ Figure 5(A) suggests that $100 \mathrm{mM} \mathrm{LiCl}\left(\kappa^{-1}=0.74 \mathrm{~nm}\right)$ is sufficient to completely screen double layer repulsions. Apparently, the low-shear viscosity is not sensitive to the residual electrostatic repulsions and/or solvation effects that cause the TPA data at $100 \mathrm{mM} \mathrm{LiCl}$ to deviate from hard-sphere behavior [Fig. 3(B)]. In contrast to $H_{s}^{r}, \eta_{L}$ is dominated by long-range $\mathrm{HI}^{56}$ and therefore relatively insensitive to details of the nearest-neighbor distribution. Nevertheless, the ionic strength dependence of $\eta_{L}$ is stronger than for $H_{s}^{r}$, because $\eta_{L}$ depends directly on DI while $H_{s}^{r}$ depends on DI only through the effect of DI on the microstructure as exemplified by Eq. (5).

When $0.5 \mathrm{M} \mathrm{LiCl}$ was added, the dispersions exhibited very slow gelation on a time scale of several days. This gelation process was evidenced by a slowly increasing viscosity and by immobilization (on the TPA time scale) of the tracer particles (see Fig. 4). The viscosity of the samples measured within $1 \mathrm{~h}$ after addition of $500 \mathrm{mM} \mathrm{LiCl}$ is shown in Fig. 5(A). The viscosity was higher than for samples with $100 \mathrm{mM} \mathrm{LiCl}$, indicating that attractive interactions dominate. ${ }^{53,54}$

\section{B. TPM-coated silica spheres in toluene-ethanol}

\section{Colloidal stability of TPM-silica in toluene-ethanol}

The same particles R75 and eR75 studied in DMFDMSO were also coated with TPM and dispersed in 7:3 v/v toluene-ethanol. The stability of a TPM-silica dispersion depends on a combination of steric stabilization afforded by the TPM layer (requiring a good, i.e., apolar, solvent for TPM) and electrical double layer repulsions (requiring a polar medium). In the apolar solvent toluene $(\varepsilon \sim 2)$, the TPM-silica particles sedimented within $1 \mathrm{~min}$, indicating a net attraction between the particles in this solvent. In the more polar solvent ethanol $(\varepsilon=25)$, TPM-silica was completely stable, as evidenced by normal sedimentation behavior and absence of any flocculation. In the optically matching 7-3 v/v toluene-ethanol mixture the particles also appeared stable by visual inspection.

These visual observations were confirmed by measuring rotational diffusion coefficients $D_{0}^{r}$ of eR75-TPM tracers in toluene-ethanol mixtures with different compositions, using TPA. Rotational diffusion should be very sensitive to any kind of aggregation since $D_{0}^{r}$ is inversely proportional to the particle volume. ${ }^{57}$ Any changes in $D_{0}^{r} \eta_{0}=k T / 8 \pi a_{T}^{3}$ with solvent composition point to changes in the radius $a_{T}$ of the diffusing species. Figure 6 shows that $D_{0}^{r} \eta_{0}$ remains constant $\left(\sim 0.22\right.$, i.e., $\left.a_{T}=90 \mathrm{~nm}\right)$ up to $\phi_{\text {toluene }}=0.84$. For $\phi_{\text {toluene }}>0.84$ there is a sharp decrease in $D_{0}^{r} \eta_{0}$ corresponding to a sharp increase in $a_{T}$. For $\phi_{\text {toluene }} \geqslant 0.9$ the eR75TPM spheres rapidly sedimented, leaving a supernatant with some remaining clusters of particles. Rotational diffusion of these clusters was completely frozen on the experimental 


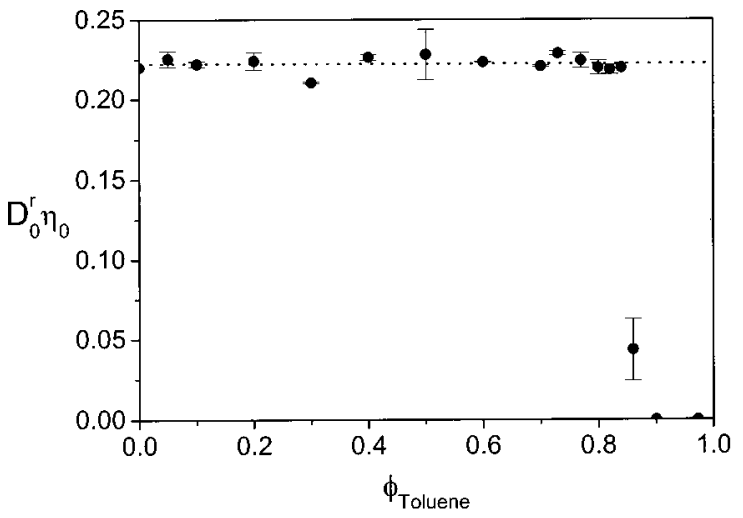

FIG. 6. Rotational self-diffusion coefficient $D_{0}^{r}$ of eR75-TPM tracers in toluene-ethanol as a function of toluene volume fraction $\phi_{\text {Toluene }}$, multiplied by the solvent viscosity $\eta_{0}$. Diffusion abruptly slows down around $85 \%$ toluene, indicating that the TPM-silica dispersion becomes unstable.

time scale $(10 \mathrm{~ms})$. We can safely conclude that for $\phi_{\text {toluene }}$ $=0.7$, which was used in all experiments (and also by previous workers), ${ }^{58-60}$ the TPM particles are sufficiently stabilized by steric and double-layer repulsions. In addition, refractive index matching in this mixture should minimize van der Waals attractions.

\section{TPA measurements of short-time rotational tracer diffusion}

Figure 7 shows reduced short-time rotational diffusion coefficients $H_{s}^{r}$ of eR75-TPM particles dispersed in suspensions of R75-TPM host spheres in toluene-ethanol. It can be seen that, just like for the uncoated particles in DMSODMF, salt addition leads to slower rotational diffusion. The data corresponding to $c_{\mathrm{LiNO}_{3}}=0$ and $1 \mathrm{mM}$ lie somewhat above the theoretical prediction Eq. (6) for hard spheres (drawn line). For $c_{\mathrm{LiNO}_{3}}=10 \mathrm{mM}$ diffusion was significantly slower than the hard-sphere prediction, suggesting an attractive interaction potential. This $\mathrm{LiNO}_{3}$ concentration is similar to the onset of attractions found in sedimentation measurements for larger TPM-silica spheres (radius $360 \mathrm{~nm}$ ) in pure ethanol. ${ }^{61}$ There, it was reported that adding $2 \mathrm{mM}$

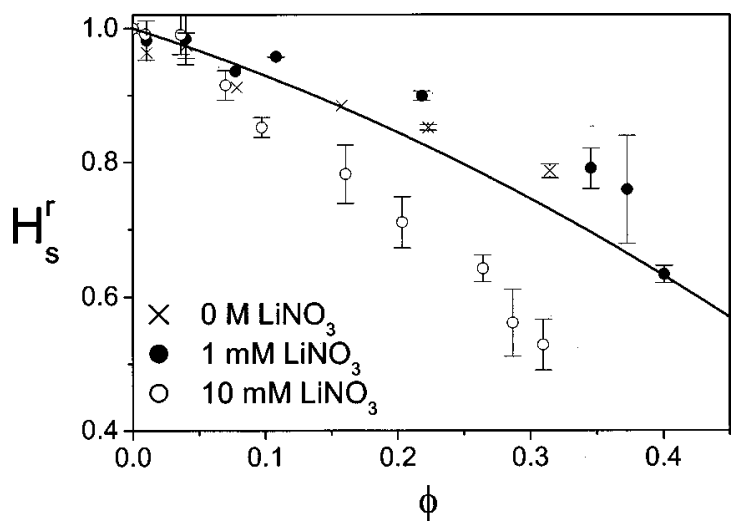

FIG. 7. Reduced short-time rotational diffusion coefficients $H_{s}^{r}$ of eR75TPM tracer spheres in R75-TPM host suspensions in 7:3 v/v tolueneethanol with various added $\mathrm{LiNO}_{3}$ concentrations as indicated. The solid line represents Eq. (6) for $H_{s}^{r}$ of hard spheres.
$\mathrm{LiNO}_{3}$ accelerated particle sedimentation relative to hard spheres, while at concentrations $c_{\mathrm{LiNO}_{3}}>4 \mathrm{mM}$ redispersion of the sediments was impossible. Both phenomena are clear signs that attractive particle interactions dominate. Attractions between our R75-TPM and eR75-TPM spheres with $10 \mathrm{mM} \mathrm{LiNO}{ }_{3}$ were, however, weak judging from the visual appearance of the samples. No flocs were observed, and sediments were easily redispersed.

\section{Rheology of host suspensions}

Like the bare silica particles in DMSO-DMF, the TPMsilica particles in toluene-ethanol displayed rheological behavior typical of charged particles [cf. Fig. 5(B)]. With increasing ionic strength the viscosity decreased due to increased electrostatic screening. At all three $\mathrm{LiNO}_{3}$ concentrations studied, the viscosity was higher than the viscosity calculated for hard spheres ${ }^{22}$ (drawn line).

At $c_{\mathrm{LiNO}_{3}}=0$ and $1 \mathrm{mM}$ the deviation of the viscosity from the hard-sphere prediction is clearly due to electrostatic repulsions, since adding more salt causes a further reduction of the viscosity. This confirms the TPA results (Fig. 7). However, from the viscosity data it remains ambiguous whether at the highest concentration of $\mathrm{LiNO}_{3}(10 \mathrm{mM})$ the deviation from hard-sphere behavior is due to repulsive or to attractive interactions. In contrast, the TPA measurements (Fig. 7) unambiguously demonstrated that at $10 \mathrm{mM} \mathrm{LiNO}_{3}$ (weak) attractive interactions dominate, since diffusion is markedly slower than the hard-sphere prediction.

\section{Generalized SED relation between rotational tracer diffusion and host suspension viscosity}

\section{Comparison between rotational tracer diffusion and host suspension viscosity}

Rotational tracer diffusion obeys a generalization of the SED relation Eq. (1) when

$$
D_{s}^{r} \eta_{\infty} / D_{0}^{r} \eta_{0}=1\left(t \ll \tau_{0}^{I}\right),
$$

or

$$
D_{L}^{r} \eta_{L} / D_{0}^{r} \eta_{0}=1\left(t \gg \tau_{0}^{I}\right) .
$$

For short times $\left(t \ll \tau_{0}^{I}\right)$ this criterion contains the highfrequency-limiting viscosity $\eta_{\infty}$, which reflects the bulk dissipation due to a high-frequency low amplitude shear oscillation of the dispersion in the linear viscoelastic regime. Like $D_{s}^{r}, \eta_{\infty}$ depends directly only on HI, while thermodynamic forces (DI and Brownian motion) enter only indirectly as they determine the equilibium microstructure. For long times $\left(t \gg \tau_{0}^{I}\right.$ ) Eq. (16) relates the long-time rotational diffusion coefficient $D_{L}^{r}$ to the zero shear viscosity $\eta_{L}$, determined by a steady-shear experiment. Both quantities refer to particle mobility in a dispersion slightly disturbed from equilibrium either by the diffusing tracer $\left(D_{L}^{r}\right)$ or by an external flow $\left(\eta_{L}\right)$, and are therefore affected directly by HI and thermodynamic forces.

This work concentrates on the short-time regime, so it would be most appropriate to compare $H_{s}^{r}$ with $\eta_{\infty}$. However, measurement of $\eta_{\infty}$ typically requires large volumes $(\geqslant 20 \mathrm{~mL}$ ), which were unfortunately not available to us due 


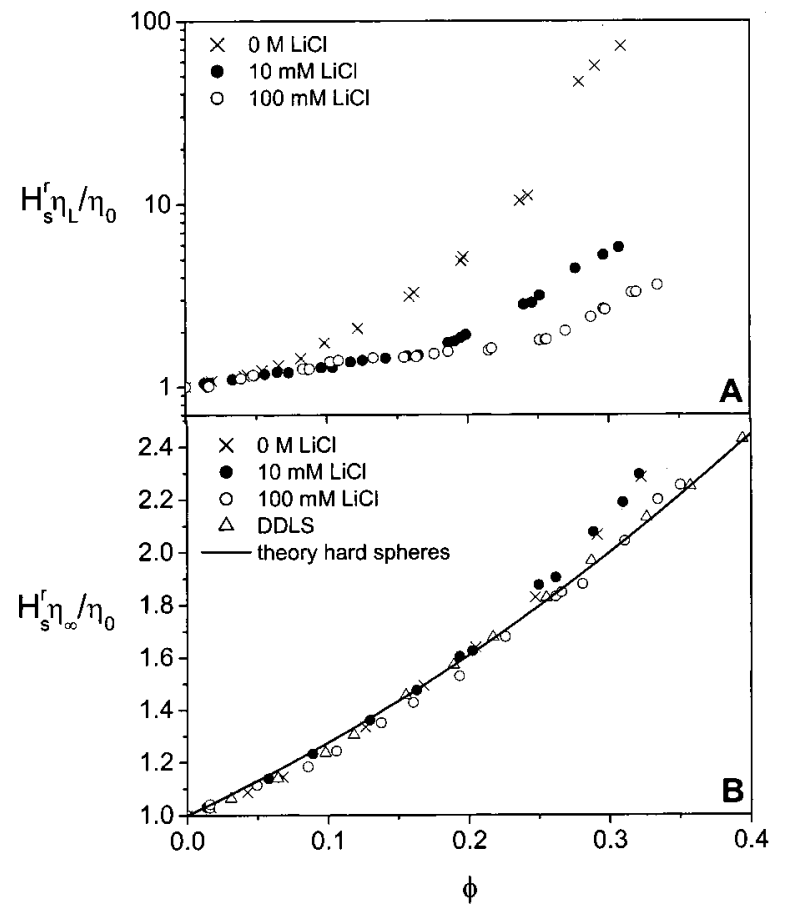

FIG. 8. SED product of $H_{s}^{r}$ (TPA data for silica in DMSO-DMF) with (A) the experimental reduced low-shear-limiting viscosity $\eta_{L} / \eta_{0}$, and (B) the high-frequency-limiting viscosity $\eta_{\infty} / \eta_{0}$ of hard spheres according to Eq. (17). Open triangles represent DDLS (Ref. 2) results for $H_{s}^{r}$ multiplied by $\eta_{\infty} / \eta_{0}$ of hard spheres according to Eq. (17). The drawn line represents the theoretical prediction for $H_{s}^{r} \eta_{\infty} / \eta_{0}$ of hard spheres calculated from Eqs. (6) and (17).

to the small scale of our model-colloid synthesis. Therefore, we compare $H_{s}^{r}$ with experimental low-shear viscosities $\eta_{L}$ and with a semiempirical expression for $\eta_{\infty}$ of hard spheres derived by Lionberger and Russel ${ }^{62}$

$$
\frac{\eta_{\infty}}{\eta_{0}}=\frac{1+\frac{3}{2} \phi\left(1+\phi-0.189 \phi^{2}\right)}{1-\phi\left(1+\phi-0.189 \phi^{2}\right)} .
$$

For hard spheres, $\eta_{\infty} \approx \eta_{L}$ up to $\phi=20 \%$. At higher volume fractions $\eta_{L} / \eta_{\infty}$ increases almost exponentially to 2.2 at $\phi$ $=40 \%$ because of the contribution of thermodynamic forces to $\eta_{L} \cdot{ }^{62,22}$ For charged spheres it is known from theory ${ }^{52}$ and an abundance of experiments that $\eta_{L}$ increases strongly with decreasing ionic strength. This was confirmed by our measurements on silica in DMSO-DMF and toluene-ethanol (Fig. 8). Measurements of $\eta_{\infty}$, especially for charged spheres, are relatively scarce. In recent experiments on sulfonated polystyrene spheres in aqueous dispersions with radii $R=60-155 \mathrm{~nm}$ and $\kappa^{-1}=1-30 \mathrm{~nm}, \eta_{\infty}$ for charged spheres was indistinguishable from that of hard spheres. ${ }^{63}$ The reason for the weak dependence of $\eta_{\infty}$ on ionic strength is first that $\eta_{\infty}$ depends only indirectly on DI, and second that $\eta_{\infty}$ is dominated by long-range $\mathrm{HI}$ and therefore not very sensitive to the microstructure. Therefore, it seems in our case a good strategy to compare the short-time diffusion results at all ionic strengths to $\eta_{\infty}$ of hard spheres. Since the actual $\eta_{\infty}$ of charged spheres is larger than or equal to that of hard spheres, the product of $H_{s}^{r}$ with the hard-sphere $\eta_{\infty}$ will underestimate the real product.

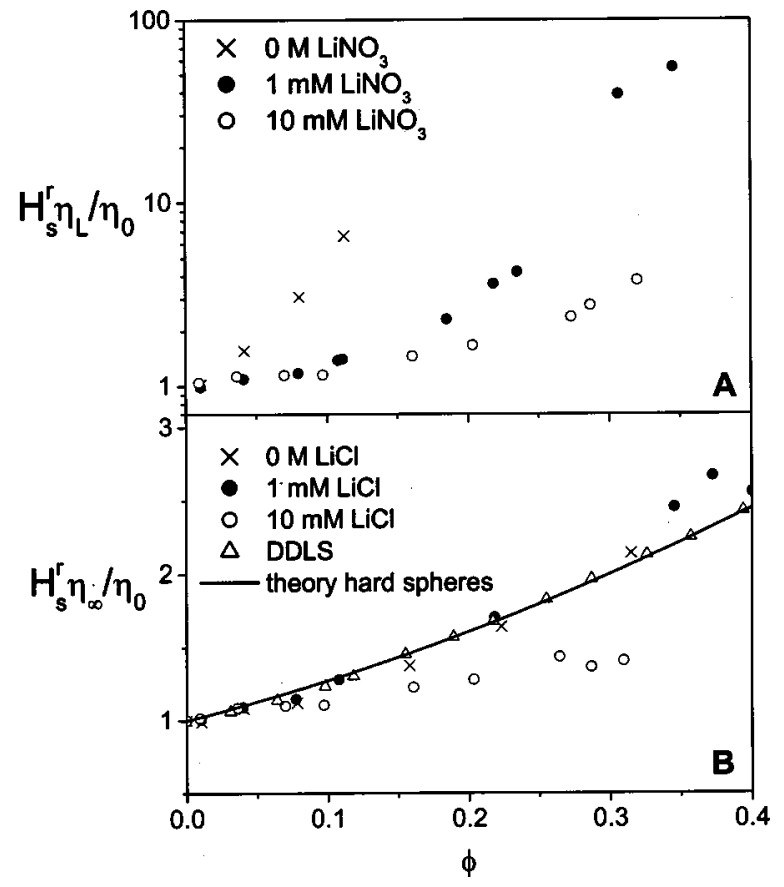

FIG. 9. SED product of $H_{s}^{r}$ (TPA data for TPM-coated silica in tolueneethanol) with (A) the experimental reduced low-shear-limiting viscosity $\eta_{L} / \eta_{0}$, and (B) the high-frequency-limiting viscosity $\eta_{\infty} / \eta_{0}$ of hard spheres according to Eq. (17). Open triangles and drawn line in (B) same as in Fig. 8(B).

Figure 8(A) shows the product of experimental data for $H_{s}^{r}(\phi)$ of eR75 tracers and for $\eta_{L}(\phi) / \eta_{0}$ of R75 host dispersions in DMSO-DMF. Large deviations from the SED product $H_{s}^{r} \eta_{L}(\phi) / \eta_{0}=1$ are seen, especially at large $\phi$ and low ionic strengths. The large difference between the three data sets almost vanishes when the experimental $H_{s}^{r}$ values are multiplied by $\eta_{\infty} / \eta_{0}$ of hard spheres according to Eq. (17) [see Fig. 8(B)]. For comparison the drawn line shows the theoretical short-time SED prediction $H_{s}^{r} \eta_{\infty} / \eta_{0}$ of hard spheres, obtained by combining Eq. (6) for $H_{s}^{r}$ and Eq. (17) for $\eta_{\infty}$. The data for $c_{\mathrm{LiCl}}=0$ and $10 \mathrm{mM}$ consistently lie somewhat above this theoretical prediction, while the data for $c_{\mathrm{LiCl}}=100 \mathrm{mM}$ are in close agreement with theory. For comparison, Fig. 8(B) also includes DDLS results for $H_{s}^{r}$ by Degiorgio et $a l^{2}{ }^{2}$ (open triangles) for teflon spheres in water containing $100 \mathrm{mM} \mathrm{NaCl}$, multiplied by Eq. (17) for $\eta_{\infty} / \eta_{0}$ of hard spheres. These data are in very good agreement with theory.

Figures 9(A) and 9(B) are the equivalents of Figs. 8(A) and $8(\mathrm{~B})$, showing data for TPM-coated silica spheres in toluene-ethanol. In Fig. 9(A), large positive deviations from $H_{s}^{r} \eta_{L}(\phi) / \eta_{0}=1$ are again seen, which decrease as the ionic strength increases or $\phi$ decreases. Figure $9(\mathrm{~B})$ shows that multiplying the experimental $H_{s}^{r}$ values by Eq. (17) for $\eta_{\infty} / \eta_{0}$ of hard spheres brings the data sets for 0 and $1 \mathrm{mM}$ $\mathrm{LiNO}_{3}$ close to the hard-sphere prediction of $H_{s}^{r} \eta_{\infty} / \eta_{0}$. However, the data for $10 \mathrm{mM} \mathrm{LiNO}_{3}$ fall below the theory, consistent with Fig. 7, where rotational diffusion is clearly significantly slower than expected for hard spheres. This is a signature of (weak) attractive interactions. 


\section{Interpretation of deviations from Stokes-Einstein-Debye scaling}

Figures 8(B) and 9(B) show that short-time SED scaling between $H_{s}^{r}$ and the high-frequency-limiting frequency viscosity $\eta_{\infty}$ is qualitatively good to within a factor of 2 for hard spheres. For charged spheres, deviations from the shorttime SED relation $H_{s}^{r} \eta_{\infty} / \eta_{0}$ are likely to be close to the deviations observed for hard spheres. This assertion is based on the fact that both $\eta_{\infty} / \eta_{0}{ }^{63}$ and $H_{s}^{r}$ [Fig. 3(A)] depend fairly weakly on double-layer interactions.

Deviations observed from generalized SE (translation) (cf. Refs. 18-22) and SED (rotation) relations for dense colloidal systems are remarkably small given the severe assumptions used in deriving the Stokes friction factors $f_{0}^{t}$ $=6 \pi \eta_{0} a_{T}$ in Eq. (2) and $f_{0}^{r}=8 \pi \eta_{0} a_{T}^{3}$ in Eq. (1). The hydrodynamic Stokes theory should strictly apply only to diffusing tracers that are much larger than the molecules comprising the liquid, as is the case for a massive colloidal particle in a molecular liquid. Obviously, this continuum assumption is questionable when the tracer diffuses through a solvent of comparable molecular mass and size. Surprisingly however, the SE(D) relations reproduce even experimental data on molecular diffusion remarkably well, in particular when the friction factors are slightly modified to ${ }^{64,65}$

$$
f_{0}^{t}=6 \nu_{0}^{t} \pi \eta_{0} a_{T}
$$

and

$$
f_{0}^{r}=8 \nu_{0}^{r} \pi \eta_{0} a_{T}^{3},
$$

introducing apparent slip parameters $\nu_{0}^{t}$ and $\nu_{0}^{r}$. In the case of perfect stick boundary conditions for the fluid velocity on the surface of the suspended tracer, $\nu_{0}^{t}$ and $\nu_{0}^{r}$ both equal one. Perfect stick assumes that the first layer of solvent sticks to the moving tracer, retarding its motion through the bulk viscosity. In the case of perfect slip boundary conditions, the tracer carries none of the surrounding liquid with it, so that friction is due only to the solvent which must be displaced to allow tracer motion. This reduces $\nu_{0}^{t}$ to $2 / 3$ and $\nu_{0}^{r}$ to zero. Experimental data on molecular diffusion are usually consistent with $\nu_{0}^{t}=2 / 3$ (perfect slip) for translation and $0<\nu_{0}^{r}$ $<1$ (partial slip) for rotation. ${ }^{65,66}$

Recently, a similar introduction of apparent slip parameters was suggested to rationalize deviations from SE scaling for tracer translational diffusion in a colloidal host fluid. ${ }^{23}$ The apparent Stokes friction factor $f^{t}(\phi)$ experienced by the tracer was written as a sum of the Stokes friction $f_{0}^{t}$ due to solvent plus friction due to neighboring host particles. There is no reason to expect a no-slip boundary condition for the host particles. Therefore, the total friction can be written as $f_{s}^{t}(\phi)=6 \pi \eta_{0} a_{T}\left[1+\nu_{s}^{t} \Delta \eta_{\infty}(\phi)\right]$ at short times and $f_{L}^{t}(\phi)$ $=6 \pi \eta_{0} a_{T}\left[1+\nu_{L}^{t} \Delta \eta_{L}(\phi)\right]$ at long times, with apparent slip parameters $\nu_{s}^{t} \in[2 / 3,1], \nu_{L}^{t} \in[0,1]$. Similarly, the reduced viscosity can be written as a sum of the solvent viscosity plus an excess part: $\eta_{\infty} / \eta_{0}=1+\Delta \eta_{\infty}$ and $\eta_{L} / \eta_{0}=1+\Delta \eta_{L}$. Values for $\nu_{s}^{t}$ and $\nu_{L}^{t}$ can be deduced from experimental data by plotting

$$
\nu_{s}^{t}=\frac{D_{0}^{t} / D_{s}^{t}-1}{\eta_{\infty} / \eta_{0}-1}
$$
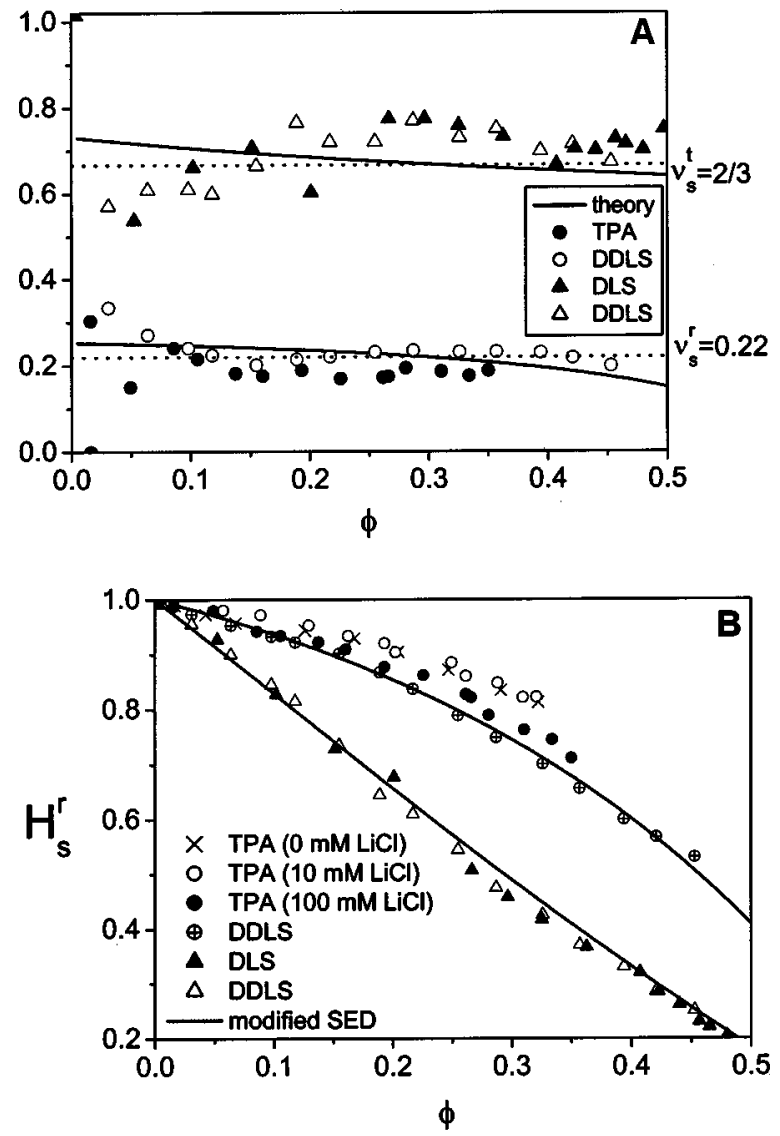

FIG. 10. (A) Apparent slip coefficients $\nu_{s}^{r}$ and $\nu_{s}^{t}$ deduced from Eqs. (19) and (20) for rotational and translational short-time tracer diffusion in host sphere suspensions. Data points are derived from experimental data obtained with TPA $\left(H_{s}^{r}\right.$, this work), DDLS (Ref. 2) $\left(H_{s}^{r}\right)$, DDLS (Ref. 2) $\left(H_{s}^{t}\right)$, and DLS (Ref. 67) $\left(H_{s}^{t}\right)$, and Eq. (17) for $\eta_{\infty} / \eta_{0}$ of hard spheres. The drawn lines are the theoretical predictions for hard spheres based on Eqs. (6) and (17) and on a semiempirical expression (Ref. 68) for $H_{s}^{t}$. The dotted lines represent overall average values $\nu_{s}^{r}=0.22$ and $\nu_{s}^{t}=2 / 3$. (B) $H_{s}^{r}$ and $H_{s}^{t}$ in the hard-sphere limit. Data points correspond to experimental TPA, DDLS (Ref. 2), and DLS (Ref. 67) results. The drawn lines represent the modified SED prediction (see text) with $\nu_{s}^{r}=0.22$ and $\nu_{s}^{t}=0.67$, and $\eta_{\infty} / \eta_{0}$ as in Eq. (17).

and

$$
\nu_{L}^{t}=\frac{D_{0}^{t} / D_{L}^{t}-1}{\eta_{L} / \eta_{0}-1}
$$

Figure 10(A) shows a plot of $\nu_{s}^{t}$ for hard spheres using for $\eta_{\infty} / \eta_{0}$ Eq. (17) and for $H_{s}^{t}$ experimental DDLS ${ }^{2}$ and DLS ${ }^{67}$ results and a semiempirical expression of Lionberger and Russel $^{68}$ which describes $H_{s}^{t}$ well up to random close packing. As seen, $\nu_{s}^{t}$ only weakly depends on $\phi$, so that the overall average value $\nu_{s}^{t} \approx 2 / 3$ gives a reasonable estimate in the full fluid regime $\phi<0.5$. Figure 10(B) shows that the modified SE relation $D_{s}^{t}(\phi)=k_{B} T / 6 \pi \eta_{0} a_{T}\left[1+\nu_{s}^{t} \Delta \eta_{\infty}(\phi)\right]$ with $\eta_{\infty}$ according to Eq. (17) is in good agreement with experimental DDLS ${ }^{2}$ and DLS ${ }^{67}$ results. For the long-time regime, Imhof $e t a l .^{23}$ found $\nu_{L}^{t} \approx 0.5$ for $\phi=0.1-0.45$, using charged silica spheres in DMF, while Segrè et al. ${ }^{21}$ reported a monotonic decrease of $\nu_{1}^{t}$ from 1 at small $\phi$ to $\phi=0.5$ for hard spheres. 
Motivated by the succes of this heuristic approach for translational diffusion, we attempt a similar analysis for our rotational diffusion data. Figure 10(A) shows a plot of $\nu_{s}^{r}$ calculated according to

$$
\nu_{s}^{r}=\frac{D_{0}^{r} / D_{s}^{r}-1}{\eta_{\infty} / \eta_{0}-1}
$$

with $\eta_{\infty} / \eta_{0}$ as in Eq. (17) and $H_{s}^{r}$ from TPA (this work), $\mathrm{DDLS}^{2}$ and the second-order virial expression in Eq. (6). The overall average value of $\nu_{s}^{r}$ in the range $\phi=0-0.5$ is 0.22 . The modified SED relation $D_{s}^{r}(\phi)=k_{B} T / 8 \pi \eta_{0} a_{T}[1$ $\left.+\nu_{s}^{r} \Delta \eta_{\infty}(\phi)\right]$ with $\eta_{\infty} / \eta_{0}$ as in Eq. (17) and $\nu_{s}^{r}=0.22$ is in fair agreement with the experimental data obtained with TPA and DDLS, ${ }^{2}$ at least in the hard-sphere limit.

We emphasize that the concept of apparent slip parameters primarily provides a qualitative picture to rationalize deviations from ideal SE(D) scaling. Actually SE(D) scaling cannot be exact for arbitrary $\phi$, since the leading order low density forms $1+A \phi+B \phi^{2}$ are different for diffusion and inverse viscosity: $A=-0.63$ for $H_{s}^{r}$ and -1.83 for $H_{s}^{t}$, while $A=-2.5$ for $\eta_{0} / \eta_{\infty}$. Moreover, $H_{s}^{r}$ and $H_{s}^{t}$ depend on DI already in the first virial coefficient $A$, whereas DI enter into $\eta_{\infty} / \eta_{0}$ only on the $O\left(\phi^{2}\right)$ level.

\section{CONCLUSIONS}

TPA was used to study the effect of tracer-host particle interactions on rotational diffusion of tracer spheres in host sphere dispersions. Colloidal systems included bare silica spheres in a polar $(\varepsilon \approx 43)$ solvent mixture (DMSO-DMF), and silane-coated spheres in a less polar $(\varepsilon \approx 9)$ solvent mixture (toluene-ethanol). In both cases the particles acquire a negative charge. Our experimental results clearly demonstrate that rotational diffusion in colloidal sphere dispersions is very sensitive to the nature of the interaction potential. Electrostatic repulsions were shown to enhance rotational diffusion (in agreement with theory), while even fairly weak attractions slow down rotation. Further, we conclude that rotational diffusion is more suited for characterizing particle interactions, especially at short ranges, than the dispersion viscosity. Namely, rotational diffusion is more sensitive to interactions near contact and can therefore detect even small deviations from a hard-sphere potential, such as residual electrostatic repulsions, solvation effects, and weak attractions. This is relevant for instance in the context of colloidal crystallization rates. ${ }^{69}$ For bare silica in DMSO-DMF at high salt $(500 \mathrm{mM} \mathrm{LiCl})$ and for TPM-silica in tolueneethanol at high toluene fractions TPA indicated particle clustering. Since rotational diffusion is very sensitive to any changes in the size of the diffusing species, TPA is in principle very suitable to monitor aggregation kinetics.

Increasing the ionic strength slows down rotational diffusion of a tracer particle, both for bare and TPM-coated silica, while it reduces the low-shear viscosity $\eta_{L}$ of the background host fluid. As a result, at low ionic strength $D_{s}^{r}$ is 1 or 2 orders of magnitude faster than predicted by the SED relation $H_{s}^{r} \eta_{L} / \eta_{0}=1$. However, with increasing ionic strength the relative deviation from SED scaling is less than a factor of 2. Scaling between $D_{s}^{r}$ and the inverse high- frequency-limiting viscosity $1 / \eta_{\infty}$ is quite accurate, though not exact, when apparent slip of the host particles on the tracer sphere surface is introduced. The physical interpretation of the dependence of the apparent slip parameter on colloid concentration and on particle interactions remains yet unclear. If the (modified) SED relation between rotational diffusion and inverse suspension viscosity is universal, diffusion measurements can be used to probe dispersion rheology. Such "microrheological" experiments are nowadays extensively used to measure viscosities of colloidal suspensions, gels, and even living cells. ${ }^{17}$ In contrast to conventional rheology, microrheology is noninvasive, requires only small sample volumes (100 $\mu \mathrm{L}$ in the case of TPA), and can measure viscosities locally in inhomogeneous samples. In view of the huge potential of microrheology for characterizing complex (biological) fluids a deeper theoretical understanding of the relation between diffusion and rheology seems to us of vital importance.

\section{ACKNOWLEDGMENTS}

The authors thank S. Sacanna, M. Pazzini, and D. van den Heuvel for preparing tracer particles; W. van der Sark and D. van den Heuvel for measuring eosin-labeling efficiencies; A. Plomp and R. Smit for conductivity experiments; J. Stolk and Y. Chong for measuring solvent densities and viscosities; and J. K. G. Dhont, C. Pathmamanoharan, G. N. Nägele, and A. Vrij for helpful discussions. This work was financially supported by The Netherlands Organization for Scientific Research (NWO/Stichting Chemische Wetenschappen).

\section{APPENDIX: ELECTROLYTE DISSOCIATION IN NONAQUEOUS SOLVENTS}

The degree of dissociation $\alpha$ of $\mathrm{LiCl}$ in DMSO-DMF and $\mathrm{LiNO}_{3}$ in toluene-ethanol, needed to calculate Debye screening lengths $\kappa^{-1}$ by Eq. (7), was estimated by measuring the molar conductivity $\Lambda$ as a function of salt concentration $c_{\mathrm{LiCl}}$ and $c_{\mathrm{LiNO}_{3}}$. Conductivities were measured using a Tetracon LF 539 electrode (WTW, cell constant $0.612 \mathrm{~cm}^{-1}$ ). Solutions were kept at $25^{\circ} \mathrm{C}$ using a thermostatic bath.

Figure 11(A) shows that $\Lambda$ decreases monotonically with $c_{\mathrm{LiCl}}$ in DMSO-DMF. The $\Lambda$ values are between those reported for $\mathrm{LiCl}$ in $\mathrm{DMF}^{70}$ and DMSO. ${ }^{71}$ The $\Lambda$ values extrapolated to $c_{\mathrm{LiCl}}=0$ are $\Lambda_{0}=80.1,35.3$, and 46.6 in units of $10^{4} \Omega \mathrm{cm}^{-2} \mathrm{~mol}^{-1}$ in DMF, DMSO, and DMSO-DMF, respectively. The product $\Lambda_{0} \eta_{0}$ is approximately constant $\left(\sim 6.4 \times 10^{-6} \mathrm{~N} \Omega \mathrm{m}^{-4} \mathrm{~s}\right)$, in agreement with the empirical Walden rule, ${ }^{72,73}$ suggesting that the conductivity difference between the different solvents is mainly due to different viscosities. At small $c_{\mathrm{LiCl}}, \Lambda$ is linear in $\sqrt{ } c_{\mathrm{LiCl}}$, which is typical of strong electrolytes. Figure 11(B) shows that $\Lambda$ for $\mathrm{LiNO}_{3}$ in ethanol also decreases monotonically with salt concentration, in agreement with previous results. ${ }^{74-76}$ Further, $\Lambda_{0}$ is $42.7 \times 10^{4} \Omega \mathrm{cm}^{-2} \mathrm{~mol}^{-1}$ and $\Lambda$ is linear in $\sqrt{ } c_{\mathrm{LiNO}_{3}}$. For $\mathrm{LiNO}_{3}$ in toluene-ethanol, however, the conductivity first decreases and then increases again, probably due to ion association into (charged) triple ions. The conductivity is much smaller than for $\mathrm{LiNO}_{3}$ in ethanol, and the concentration 
$\Lambda$
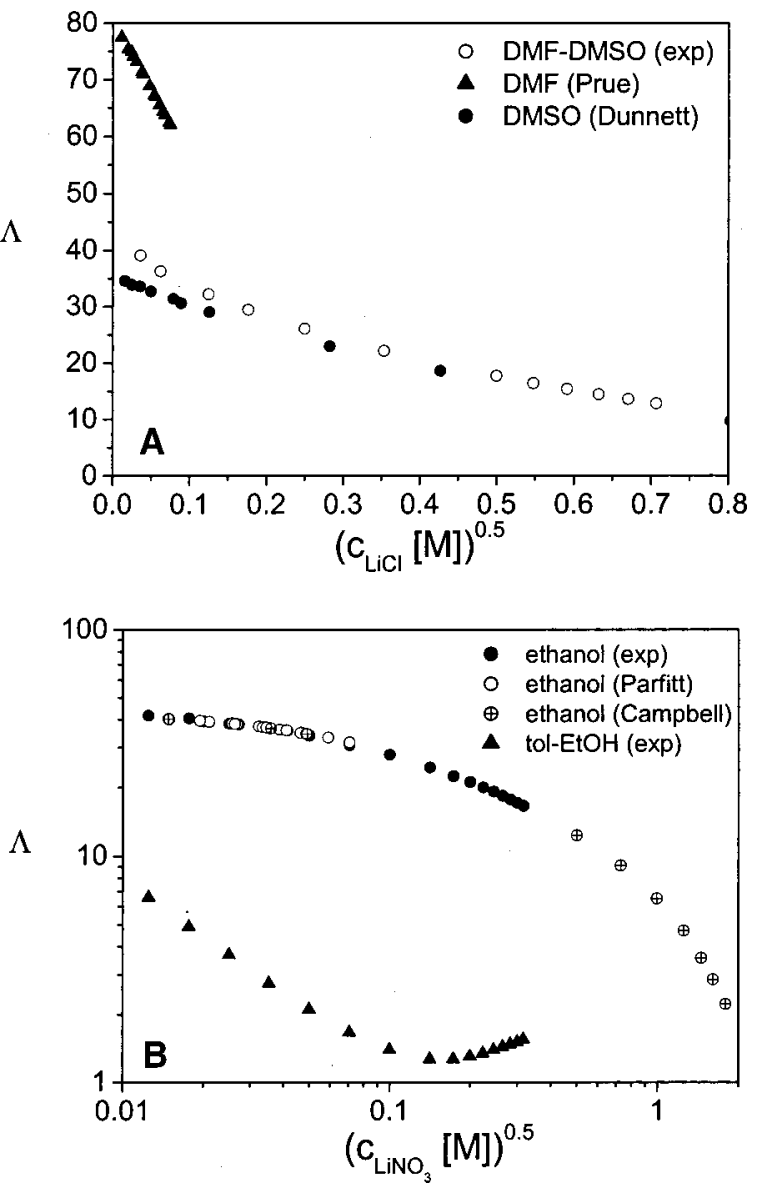

FIG. 11. Molar conductivity $\Lambda$ (in units of $10^{4} \mathrm{~m}^{2} \Omega^{-1} \mathrm{~mol}^{-1}$ ) of (A) $\mathrm{LiCl}$ in DMF, taken from Prue et al. (Ref. 70), in DMSO, taken from Dunnett et al. (Ref. 71), and in 3:2 v/v DMSO-DMF, measured in this work. (B) Same for $\mathrm{LiNO}_{3}$ in ethanol, measured in this work and taken from Campbell et al. (Ref. 75) and Parfitt et al. (Ref. 76), and in 7:3 v/v toluene-ethanol, measured in this work.

dependence at low $c_{\mathrm{LiNO}_{3}}$ is stronger than $\sqrt{ } c_{\mathrm{LiNO}_{3}}$. Further, Walden's rule is not applicable; $\Lambda_{0} \eta_{0}=4.6 \times 10^{-6}$ $\mathrm{N} \Omega \mathrm{m}^{-4} \mathrm{~s}$ in ethanol and $\sim 9 \times 10^{-7} \mathrm{~N} \Omega \mathrm{m}^{-4} \mathrm{~s}$ in tolueneethanol. All this indicates that there is significant ion association in toluene-ethanol, which can be expected in view of the small dielectric constant $(\varepsilon \sim 9)$.

The degree of dissociation $\alpha$ for fairly strong electrolytes up to concentrations of $10 \mathrm{mM}$ can be estimated from $\alpha$
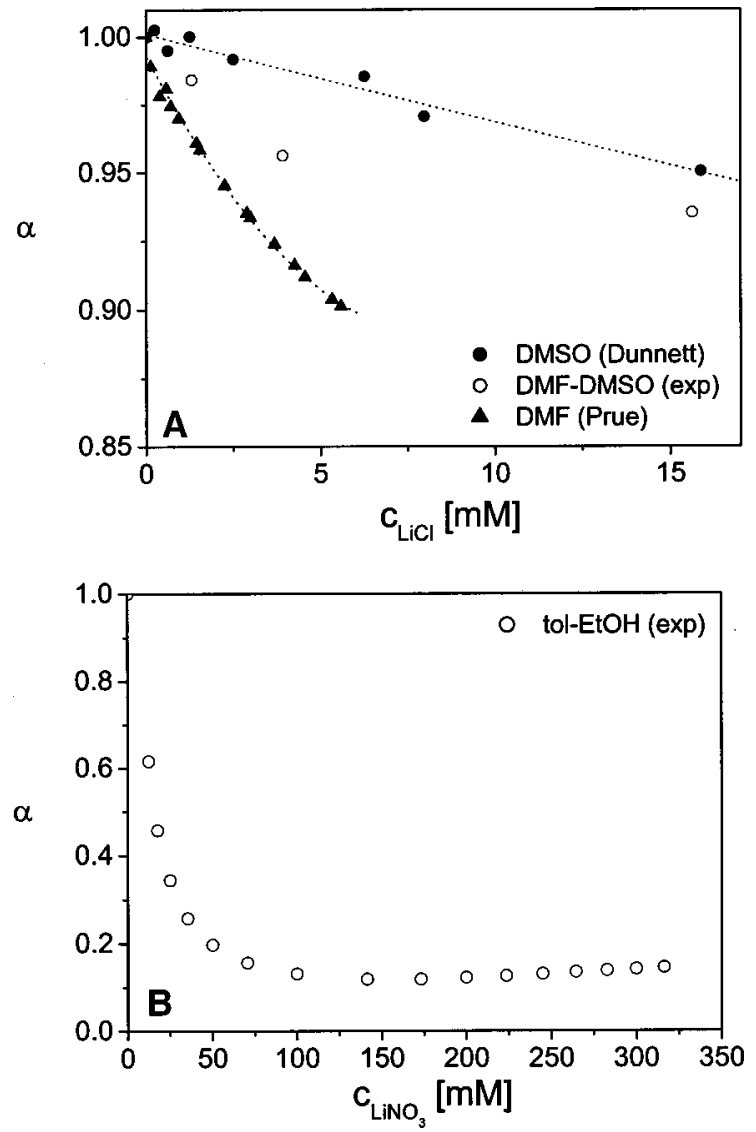

FIG. 12. Degree of dissociation $\alpha$ of (A) LiCl in DMF, DMSO, and 3:2 v/v DMSO-DMF, calculated using $\alpha=\Lambda / \Lambda_{\text {FO }}$ and Eq. (20), and of (B) $\mathrm{LiNO}_{3}$ in $7: 3 \mathrm{v} / \mathrm{v}$ toluene-ethanol, calculated using $\alpha=\Lambda / \Lambda_{0}$. The dotted lines are to guide the eye.

$=\Lambda_{\text {exp }} / \Lambda_{\mathrm{FO}}$, where $\Lambda_{\mathrm{FO}}$ is the molar conductivity calculated from the Fuoss-Onsager equation for completely dissociated electrolytes $(\alpha=1)$ :

$$
\Lambda_{\mathrm{FO}} \approx \Lambda_{0}-S \sqrt{c_{\text {salt }}}+E c_{\text {salt }} \log \left(c_{\text {salt }} / \mathrm{mol} \mathrm{L}^{-1}\right)+J c_{\text {salt }} .
$$

This equation accounts for ion interactions. We neglected higher-order terms in $c_{\text {salt }}$ and the effect of $c_{\text {salt }}$ on the viscosity. Values for $S, E$, and $J$ obtained using $\varepsilon$ and $\eta_{0}$ from Table I are collected in Table IV. Figure 12(A) shows the degree of dissociation $\alpha=\Lambda_{\exp } / \Lambda_{\mathrm{FO}}$ of $\mathrm{LiCl}$ in DMSO-

TABLE IV. Fuoss-Onsager calculations according to Eq. (A1) for $\mathrm{LiNO}_{3}$ in ethanol and 7:3 v/v tolueneethanol (Tol-EtOH) and for $\mathrm{LiCl}$ in DMF, DMSO, and 3:2 v/v DMSO-DMF.

\begin{tabular}{lcccc}
\hline \hline \multicolumn{1}{c}{ Solvent } & $\begin{array}{c}\Lambda_{0} / \\
\left(\mathrm{cm}^{2} \Omega^{-1} \mathrm{~mol}^{-1}\right)\end{array}$ & $\begin{array}{c}S^{\mathrm{a}} \\
\left(\mathrm{cm}^{2} \Omega^{-1} \mathrm{~mol}^{-3 / 2} \mathrm{dm}^{3 / 2}\right)\end{array}$ & $\begin{array}{c}E l^{\mathrm{b}} \\
\left(\mathrm{cm}^{2} \Omega^{-1} \mathrm{~mol}^{-2} \mathrm{dm}^{3}\right)\end{array}$ & $\begin{array}{c}J / /^{\mathrm{c}} \\
\left(\mathrm{cm}^{2} \Omega^{-1} \mathrm{~mol}^{-2} \mathrm{dm}^{3}\right)\end{array}$ \\
\hline Ethanol & 42.7 & 141.1 & 164.2 & 147.4 \\
Tol-EtOH & 10.7 & 308.1 & -260.3 & 95.24 \\
DMF & 80.1 & 151.6 & 85.20 & 208.3 \\
DMSO & 35.3 & 51.21 & 16.83 & 135.3 \\
DMSO-DMF & 46.6 & 79.57 & 26.51 & 153.7 \\
\hline \hline
\end{tabular}

${ }^{\mathrm{a}} S=S_{1} \Lambda_{0}+S_{2}$ with $S_{1}=8.2043 \times 10^{5} /(\varepsilon T)^{3 / 2}$ and $S_{2}=8.2484 /(\eta \sqrt{\varepsilon T})$.

${ }^{\mathrm{b}} E=E_{1} \Lambda_{0}-E_{2}$ with $E_{1}=2.94227 \times 10^{12} /(\varepsilon T)^{3}, E_{2}=8.66408 \times 10^{6} /\left(\eta(\varepsilon T)^{2}\right)$.

${ }^{\mathrm{c}} J=J_{1} \Lambda_{0}+J_{2}$ with $J_{1}=0.4582[h(b)+\ln [\dot{a}]-0.0941]$ and $J_{2}=15.48+18.15 \dot{a}-17.66 \ln [\dot{a}]$, with $h(b)=\left(2 b^{2}\right.$

$+2 b-1) / b^{3}$ and $b=7.135 / \dot{a}$, where $\dot{a}$ represents the distance of closest approach between two ions (in units of $10^{-10} \mathrm{~m}$ ) which we set equal to 5 . 
DMF, DMSO, and DMF thus calculated. In all three solvents $\alpha$ is close to one. In DMSO-DMF $\alpha$ lies between that in DMF and DMSO. Dissociation is highest in DMSO, which happens to have the largest dielectric constant. For $\mathrm{LiNO}_{3}$ in ethanol dissociation is near complete (result not shown). For $\mathrm{LiNO}_{3}$ in toluene-ethanol we did not obtain consistent results using Eq. (A1). Since $\mathrm{LiNO}_{3}$ behaves as a weak electrolyte the decrease of $\Lambda$ with increasing $c_{\text {salt }}$ is probably dominated by ion pairing. Neglecting ion interactions, $\Lambda$ is then described by the Arrhenius law $\alpha=\Lambda / \Lambda_{0}$. Figure 12(B) shows the $c_{\mathrm{LiNO}_{3}}$ dependence of $\alpha$ thus estimated. For salt concentrations above $10 \mathrm{mM}$, Eq. (A1) breaks down, so it becomes difficult to evaluate $\alpha$ from conductivity experiments. Therefore, we set for simplicity $\alpha\left(c_{\mathrm{LiCl}}>10 \mathrm{mM}\right)$ $=0.9$ for DMSO-DMF and $\alpha\left(c_{\mathrm{LiNO}_{3}}>10 \mathrm{mM}\right)=0.14$ for toluene-ethanol.

${ }^{1}$ J. K. G. Dhont, An Introduction to Dynamics of Colloids (Elsevier, Amsterdam, 1996).

${ }^{2}$ V. Degiorgio, R. Piazza, and R. B. Jones, Phys. Rev. E 52, 2707 (1995).

${ }^{3}$ G. H. Koenderink and A. P. Philipse, Langmuir 16, 5631 (2000).

${ }^{4}$ A. Tolle and H. Sillescu, Langmuir 10, 4420 (1994).

${ }^{5}$ D. Geschke and G. Fleischer, Z. Phys. Chem. (Munich) 190, 41 (1995).

${ }^{6}$ G. A. Barrall, K. Schmidt-Rohr, Y. K. Lee, K. Landfester, H. Zimmermann, G. C. Chingas, and A. Pines, J. Chem. Phys. 104, 509 (1996).

${ }^{7}$ J. Kanetakis, A. Tolle, and H. Sillescu, Phys. Rev. E 55, 3006 (1997).

${ }^{8}$ J. Kanetakis and H. Sillescu, Chem. Phys. Lett. 252, 127 (1996).

${ }^{9}$ M. Velez and D. Axelrod, Biophys. J. 53, 575 (1988).

${ }^{10}$ M. P. Lettinga, C. M. van Kats, and A. P. Philipse, Langmuir 16, 6166 (2000).

${ }^{11}$ M. P. Lettinga, M. A. M. J. van Zandvoort, C. M. van Kats, and A. P. Philipse, Langmuir 16, 6156 (2000).

${ }^{12}$ G. H. Koenderink, H. Zhang, M. P. Lettinga, G. Nägele, and A. P. Philipse, Phys. Rev. E 64, 022401 (2001).

${ }^{13}$ D. Quemada and C. Berli, Adv. Colloid Interface Sci. 98, 51 (2002).

${ }^{14}$ G. Stokes, Trans. Cambridge Philos. Soc. 9, 5 (1856).

${ }^{15}$ A. Einstein, Ann. Phys. (Leipzig) 19, 371 (1906).

${ }^{16}$ P. Debye, Polar Molecules (Dover, New York, 1929).

${ }^{17}$ T. Gisler and D. A. Weitz, Curr. Opin. Colloid Interface Sci. 3, 586 (1999).

${ }^{18}$ M. M. Kops-Werkhoven and H. M. Fijnaut, J. Chem. Phys. 77, 2242 (1982).

${ }^{19}$ C. W. J. Beenakker, Physica A 128, 48 (1984).

${ }^{20}$ A. van Blaaderen, J. Peetermans, G. Maret, and J. K. G. Dhont, J. Chem. Phys. 96, 4591 (1992)

${ }^{21}$ P. N. Segrè, S. P. Meeker, P. N. Pusey, and W. C. K. Poon, Phys. Rev. Lett. 75, 958 (1995).

${ }^{22}$ A. J. Banchio, G. Nägele, and J. Bergenholtz, J. Chem. Phys. 111, 8721 (1999).

${ }^{23}$ A. Imhof, A. van Blaaderen, G. Maret, and J. G. K. Dhont, J. Chem. Phys. 100, 2170 (1994).

${ }^{24}$ J. Bergenholtz, F. M. Horn, W. Richtering, N. Willenbacher, and N. J. Wagner, Phys. Rev. E 58, R4088 (1998).

${ }^{25}$ M. Watzlawek and G. Nägele, Physica A 235, 56 (1997).

${ }^{26}$ R. B. Jones, Physica A 157, 752 (1989).

${ }^{27}$ B. U. Felderhof and R. B. Jones, Phys. Rev. E 48, 1084 (1993).

${ }^{28}$ B. U. Felderhof and R. B. Jones, Phys. Rev. E 48, 1142 (1993).

${ }^{29}$ R. B. Jones, Physica A 150, 339 (1988).

${ }^{30}$ B. Cichocki, M. L. Ekiel-Jezewska, and E. Wajnryb, J. Chem. Phys. 111, 3265 (1999)
${ }^{31} \mathrm{H}$. Zhang, and G. Nägele (unpublished).

${ }^{32} \mathrm{H}$. Zhang and G. Nägele, J. Chem Phys. (to be published).

${ }^{33}$ M. H. J. Hagen, D. Frenkel, and C. P. Lowe, Physica A 272, 376 (1999).

${ }^{34}$ A. Tulpar, V. Subramanian, and W. Ducker, Langmuir 17, 8451 (2001).

${ }^{35}$ E. J. W. Verwey and J. T. G. Overbeek, Theory of the Stability of Lyophobic Colloids (Elsevier, New York, 1948).

${ }^{36}$ R. G. Gordon, J. Chem. Phys. 45, 1643 (1966).

${ }^{37}$ T. Tao, Biopolymers 8, 609 (1969).

${ }^{38}$ T. J. Chuang and K. B. Eisenthal, J. Chem. Phys. 57, 5094 (1972).

${ }^{39}$ M. P. Lettinga, H. Zuilhof, and M. A. M. J. van Zandvoort, Phys. Chem. Chem. Phys. 2, 3697 (2000).

${ }^{40}$ A. P. Philipse and A. Vrij, J. Colloid Interface Sci. 128, 121 (1989).

${ }^{41}$ W. Stöber, A. Fink, and E. Bohn, J. Colloid Interface Sci. 26, 62 (1968).

${ }^{42}$ A. van Blaaderen and A. Vrij, Langmuir 8, 2921 (1992).

${ }^{43}$ K. Osseo-Asare and F. J. Arriagada, Colloids Surf. 50, 321 (1990).

${ }^{44}$ M. P. Lettinga, PhD thesis, Utrecht University, Utrecht, The Netherlands, 1999.

${ }^{45}$ S. Wermeille and F. Stoeckli (unpublished results, 2000).

${ }^{46}$ F. Stoeckli, D. Hugi-Cleary, and T. A. Centeno, J. Eur. Ceram. Soc. 18, 1177 (1998).

${ }^{47}$ A. Grabbe and R. G. Horn, J. Colloid Interface Sci. 157, 375 (1993).

${ }^{48}$ R. K. Iler, The Chemistry of Silica (Wiley, New York, 1979).

${ }^{49}$ T. W. Healy, Adv. Chem. Ser. 234, 147 (1994).

${ }^{50}$ J. J. Adler, Y. I. Rabinovich, and B. M. Moudgil, J. Colloid Interface Sci. 237, 249 (2001).

${ }^{51}$ G. Vigil, X. Zhenghe, S. Steinberg, and J. Israelachvili, J. Colloid Interface Sci. 165, 367 (1994).

${ }^{52}$ W. B. Russel, J. Fluid Mech. 85, 209 (1978).

${ }^{53}$ W. B. Russel, J. Chem. Soc., Faraday Trans. 2 80, 31 (1984).

${ }^{54}$ B. Cichocki and B. U. Felderhof, J. Chem. Phys. 93, 4427 (1990).

${ }^{55}$ A. T. J. M. Woutersen and C. G. de Kruif, J. Chem. Phys. 94, 5739 (1991).

${ }^{56}$ S. Kim and R. T. Mifflin, Phys. Fluids 28, 2033 (1985).

${ }^{57}$ A. Vailati, D. Asnaghi, M. Giglio, and R. Piazza, Phys. Rev. E 48, R2358 (1993).

${ }^{58}$ A. P. Philipse and A. Vrij, J. Chem. Phys. 88, 6459 (1988).

${ }^{59}$ J. S. van Duijneveldt, J. K. G. Dhont, and H. N. W. Lekkerkerker, J. Chem. Phys. 99, 6941 (1993).

${ }^{60}$ Y. D. Yan and J. K. G. Dhont, Physica A 198, 78 (1993).

${ }^{61}$ D. M. E. Thies-Weesie, A. P. Philipse, G. Nägele, B. Mandl, and R. Klein, J. Colloid Interface Sci. 176, 43 (1995).

${ }^{62}$ R. A. Lionberger and W. B. Russel, J. Rheol. 38, 1885 (1994).

${ }^{63}$ F. M. Horn, W. Richtering, J. Bergenholtz, N. Willenbacher, and N. J. Wagner, J. Colloid Interface Sci. 225, 166 (2000).

${ }^{64}$ B. J. Alder, D. M. Gass, and T. E. Wainwright, J. Chem. Phys. 53, 3813 (1970).

${ }^{65}$ C. M. Hu and R. Zwanzig, J. Chem. Phys. 60, 4354 (1974).

${ }^{66}$ J. L. Dote, D. Kivelson, and R. N. Schwartz, J. Phys. Chem. 85, 2169 (1981).

${ }^{67}$ P. N. Segrè, O. P. Behrend, and P. N. Pusey, Phys. Rev. E 52, 5070 (1995).

${ }^{68}$ R. A. Lionberger and W. B. Russel, Adv. Chem. Phys. 111, 399 (2000).

${ }^{69}$ C. Smits, J. S. van Duijneveldt, J. K. G. Dhont, and H. N. W. Lekkerkerker, Phase Transitions 21, 157 (1990).

${ }^{70}$ J. E. Prue and P. J. Sherrington, Trans. Faraday Soc. 57, 1795 (1961).

${ }^{71}$ J. S. Dunnett and R. P. H. Gasser, Trans. Faraday Soc. 61, 922 (1965).

${ }^{72}$ P. Walden, H. Ulich, and G. Busch, Z. Phys. Chem., Stoechiom. Verwandtschaftsl. 123, 429 (1926).

${ }^{73}$ P. Walden and E. I. Birr, Z. Phys. Chem. Abt. A 153, 1 (1931).

${ }^{74}$ E. D. Copley, D. M. Murray-Rust, and H. Hartley, J. Electrochem., 1930, 2492.

${ }^{75}$ A. N. Campbell and G. H. Debus, Can. J. Chem. 34, 1232 (1956).

${ }^{76}$ G. D. Parfitt and A. L. Smith, Trans. Faraday Soc. 59, 257 (1963).

${ }^{77}$ Handbook of Chemistry and Physics, edited by D. R. Lide (CRC, Boca Raton, FL, 1995). 